\title{
Magnetoelastic coupling and multiferroic ferroelastic/magnetic phase transitions in the perovskite $\mathrm{KMnF}_{3}$
}

\author{
M. A. Carpenter* and E. K. H. Salje \\ Department of Earth Sciences, University of Cambridge, Downing Street, Cambridge CB2 3EQ, United Kingdom
}

Christopher J. Howard

School of Engineering, University of Newcastle, Callaghan NSW 2308, Australia

(Received 27 March 2012; revised manuscript received 14 May 2012; published 25 June 2012)

\begin{abstract}
The magnetic, elastic, and anelastic behavior of single-crystal $\mathrm{KMnF}_{3}$ have been investigated by superconducting quantum interference device (SQUID) magnetometry and resonant ultrasound spectroscopy (RUS) through the sequence of phase transitions: phase I, $\operatorname{Pm} \overline{3} m \rightarrow\left(T_{\mathrm{c} 1}=185 \mathrm{~K}\right) \rightarrow$ phase II, $I 4 / m c m \rightarrow\left(T_{\mathrm{c} 2}=T_{\mathrm{N}}=87 \mathrm{~K}\right) \rightarrow$ phase III, antiferromagnetic, $\mathrm{Cmcm} \rightarrow\left(T_{\mathrm{c} 3}=82 \mathrm{~K}\right) \rightarrow$ phase IV, canted ferromagnet, Pnma. It is concluded that observed changes in the elastic properties can be explained simply in terms of strain/order parameter coupling for the octahedral tilting transitions. There appears to be no evidence in the present data or in data from the literature for coupling between the magnetic order parameter and shear strains. Any coupling between the magnetic and structural transitions is therefore weak, probably occurring only biquadratically through a small common volume strain. The combined data show unambiguously that, for the crystal used, the Néel point and the structural transition at $87 \mathrm{~K}$ are coincident. In other crystals, with slightly different stoichiometries and defect contents, this need not be the case, however, and the overlap of transition temperatures in $\mathrm{KMnF}_{3}$ is essentially accidental. Strong acoustic dissipation at $\sim 0.1-1 \mathrm{MHz}$ in the stability field of phase II is attributed to the local mobility of transformation twin walls under externally applied stress. A Debye-like loss peak near $130 \mathrm{~K}$ is attributed to pinning of at least some twin walls by defects, but relatively high levels of acoustic dissipation below this freezing temperature imply that some of the twin walls remain mobile due to weak pinning or the absence of any pinning. Acoustic losses continue in the stability field of phase III $(\mathrm{Cmcm})$ but diminish substantially in the stability field of phase IV (Pnma), implying quite different twin mobilities in the different structure types. Overlap of the structural and magnetic instabilities in $\mathrm{KMnF}_{3}$ opens up possibilities for manipulation of ferroelastic twinning by application of a magnetic field and for creation of materials in which the ferroelastic twin walls have quite different magnetic properties from the matrix in which they lie.
\end{abstract}

DOI: 10.1103/PhysRevB.85.224430

PACS number(s): 75.80.+q, 62.65.+k, 62.80.+f, 62.40.+i

\section{INTRODUCTION}

Interest in the sequence of structural and magnetic phase transitions in $\mathrm{KMnF}_{3}$ appears not to have declined since they were first discovered in the early 1960s. The relationship between magnetic transitions and other types of phase transitions has also acquired a more general significance given the importance of magnetoelastic coupling in the context of the properties of multiferroic materials. With the benefit of hindsight, crystallographic aspects of the transitions in $\mathrm{KMnF}_{3}$ seem to have been defined most clearly by Gibaud et al. ${ }^{1}$ who gave the sequence as phase I, $\operatorname{Pr} \overline{3} m \rightarrow\left(T_{\mathrm{c} 1}=186.5 \mathrm{~K}\right) \rightarrow$ phase II, $I 4 / m c m \rightarrow\left(T_{\mathrm{c} 2}=88 \mathrm{~K}\right) \rightarrow$ phase III, orthorhombic $\rightarrow$ $\left(T_{\mathrm{c} 3}=82 \mathrm{~K}\right) \rightarrow$ phase IV, pseudotetragonal but probably orthorhombic.

The cubic $(P m \overline{3} m) \rightarrow$ tetragonal $(I 4 / \mathrm{mcm})$ transition is weakly first order but close to tricritical in character. ${ }^{1-11}$ Values of $T_{\mathrm{c} 1}$ from the literature are in the range 184-189 K, and there are only two outliers at $195 \mathrm{~K}$ (Table I). The space groups of phases III and IV have not been determined, but single-crystal diffraction patterns from phase III reveal the presence of both $R$-point and $M$-point reflections. ${ }^{1,2,29,30,33}$ Thus, it appears that phase II arises by condensation of an $R$-point soft mode and phase III by condensation of an $M$-point soft mode, ${ }^{2,39,40}$ in the classical manner of octahedral tilting transitions in perovskites. In some diffraction data the II $\rightarrow$ III transition looks first order
(Refs. 2 and 29 and thin crystal of Ref. 30), while in others it looks continuous (Ref. 25 and thick crystal of Ref. 30); reported values of $T_{\mathrm{c} 2}$ are between 88 and $103 \mathrm{~K}$ (Table I). In marked contrast with the disparities for II $\rightarrow$ III, there is close agreement in the literature that the structural transition between phases III and IV is first order in character, with $T_{\mathrm{c} 3}$ close to $83 \mathrm{~K}$ and a hysteresis between cooling and heating of 1 to $2 \mathrm{~K}$ (e.g., Refs. 28-30 and 33). Only one reported value of $T_{\mathrm{c} 3}$ exists outside this range $\left(90.5 \mathrm{~K}^{38}\right)$. There do not appear to be overriding or systematic variations in behavior with crystal size, which vary between several millimeters thick as used for neutron diffraction (e.g., Refs. 2 and 25) to fractions of a millimeter thick as used for x-ray diffraction (e.g., Ref. 30). Subtle strain effects and/or some influence from different twin configurations may be important for II $\rightarrow$ III. $^{30}$

There has been considerable discussion in the literature about the possible space groups for the low-temperature structures, and $P 4 / \mathrm{mbm}, \mathrm{Cmcm}, P n m a, P m m n$, and $P 2_{1} / \mathrm{m}$ have all been proposed. ${ }^{15,29,32,41-43} P$ nma can be ruled out for phase III because the II $\rightarrow$ III transition in samples doped with Ca appears to be thermodynamically continuous, ${ }^{1}$ and Pnma is not a subgroup of $I 4 / \mathrm{mcm}$. In the symmetry hierarchy of Howard and Stokes, ${ }^{44}$ the only orthorhombic structure that can develop by a continuous $M$-point transition from the $I 4 / \mathrm{mcm}$ structure has space group $\mathrm{Cmcm}$. Following Beckman and Knox, ${ }^{15}$ Pnma remains the most likely space group for 
TABLE I. Transition temperatures for $\mathrm{KMnF}_{3}$ taken from the literature. Temperatures in the column for $T_{\mathrm{c} 3}$ include determinations of magnetic and structural transitions. An asterisk refers to measurements on samples carried out on crystals grown by the Bridgman technique at the Université du Maine, Le Mans, France (Gibaud et al., Ref. 1).

\begin{tabular}{|c|c|c|c|c|}
\hline Source & $T_{\mathrm{c} 3}(\mathrm{~K})$ & $T_{\mathrm{N}}(\mathrm{K})$ & $T_{\mathrm{c} 2}(\mathrm{~K})$ & $T_{\mathrm{c} 1}(\mathrm{~K})$ \\
\hline Ogawa $(1959)^{12}$ & & $\begin{array}{l}95 \pm 3 \\
89 \pm 1\end{array}$ & & \\
\hline Shulman et al. $(1959)^{13}$, in Scatturin et al. $(1961)^{14}$ & & 88.3 & & \\
\hline Beckman and Knox $(1961)^{15}$ & 81.5 & 88 & & 184 \\
\hline Heeger et al. $(1961)^{16}$ & 81.5 & 88.3 & & \\
\hline Cooper and Nathans $(1966)^{17}$ & & 88.1 & & \\
\hline Shirane et al. $(1970)^{2}$ & & & 91.5 & 186.6 \\
\hline Furukawa et al. $(1970)^{18}$ & & & & 186.7 \\
\hline Minkiewicz et al. $(1970)^{19}$ & & & & 184 \\
\hline Aleksandrov and Reshchikova $(1970)^{3}$ & & & & 195 \\
\hline Melcher and Plovnik $(1971)^{20}$ & & & & 186.2 \\
\hline Saiki $(1972)^{21}$ & 81.5 & 88.3 & & \\
\hline Maartense and Searle $(1972)^{22}$ & 82 & $87.9 \pm 0.1$ & & 184 \\
\hline Borsa $(1973)^{23}$ & & & & 186 \\
\hline Hirotsu and Sawada $(1973)^{7}$ & & & & 186 \\
\hline Fossheim et al. $(1974)^{24}$ & & & 91.5 & 187.5 \\
\hline Hidaka et al. $(1975)^{25}$ & 81 & 88 & 91 & \\
\hline Benard and Walker $(1976)^{26}$ & 80 & & 89 & 184 \\
\hline Holt and Fossheim $(1981)^{27}$ & & & & 187.2 \\
\hline Stokka et al. $(1981)^{4}$ & & & & 186.9 \\
\hline Sakashita et al. $(1981)^{5}$ & & & & 186.7 \\
\hline Bartolomé et al. $(1983)^{28}$ & $83 / 84$ & $87.7 \pm 0.1$ & 95 & 188 \\
\hline Hidaka et al. $(1986)^{29}$ & 83 & 88 & 88 & \\
\hline \multirow[t]{2}{*}{ Hidaka et al. $(1989)^{30}$} & 83 & & 92 & \\
\hline & 83 & & 88.0 & \\
\hline Sakashita et al. $(1990)^{6}$ & & & & 186 \\
\hline${ }^{*}$ Gibaud et al. $(1991)^{1}$ & 82 & & 88 & 186.5 \\
\hline Ratuszna and Kachel $(1992)^{31}$ & & & 91 & 186 \\
\hline Kapusta et al. $(1999)^{32}$ & & & 96 & 195 \\
\hline${ }^{*}$ Hayward et al. $(2000)^{11}$ & & & & 186.0 \\
\hline Watanabe et al. $(2006)^{33}$ & $81-84$ & & & \\
\hline Salazar et al. $(2007)^{34}$ & $79.5 / 81.1$ & & 86.6 & 186.2 \\
\hline${ }^{*}$ Schranz et al. $(2009)^{35}$ & & & 91.0 & 186.7 \\
\hline${ }^{*}$ Salje et al. $(2009)^{36}$ & & & & 186.0 \\
\hline${ }^{*}$ Salje and Zhang $(2009)^{37}$ & & & & 184 \\
\hline \multirow[t]{2}{*}{ Kizhaev and Markova $(2011)^{38}$} & 82.5 & 87.7 & 94.8 & 187.4 \\
\hline & 90.5 & 87.1 & 102.5 & 189.3 \\
\hline * This study: magnetic & $80 / 82$ & 87.3 & & 185 \\
\hline elastic & 83 & & 87 & 185 \\
\hline
\end{tabular}

phase IV as it is the typical low-temperature structure of most perovskites with both $R$ - and $M$-point tilting, including $\mathrm{NaMnF}_{3}$ and $\mathrm{NH}_{4} \mathrm{MnF}_{3}$ for example. ${ }^{45-47}$ Thus, from a purely structural point of view, the transition evolution appears to be $P m \overline{3} m \rightarrow I 4 / m c m \rightarrow C m c m \rightarrow$ Pnma with falling temperature. This is also the sequence proposed for Li-doped and $\mathrm{Na}$-doped $\mathrm{KMnF}_{3}$ by Ratuszna. ${ }^{41}$ The $\mathrm{I} 4 / \mathrm{mcm} \rightarrow \mathrm{Cmcm}$ transition for phase II $\rightarrow$ phase III would be driven simply by softening of the $M$-point optic mode in the manner shown by Shirane et al. ${ }^{2}$ From the evidence of lattice parameter variations in Gibaud et al., ${ }^{1}$ it appears to be weakly first order/close to tricritical in undoped crystals and tricritical in Ca-doped crystals. Cmcm $\rightarrow$ Pnma for III $\rightarrow$ IV can only be first order because it involves a change in the orientation of $R$-point tilting. Kapusta et al $^{32}$ reported evidence of line broadening in a powder diffraction pattern collected at $12 \mathrm{~K}$, which was fit with a monoclinic lattice $\left(P 2_{1} / m\right)$. This structure could not then extend up to $91 \mathrm{~K}$ as proposed, however, in view of the clear evidence for the structural transition at $83 \mathrm{~K}$.

Two magnetic transitions were originally reported in $\mathrm{KMnF}_{3}$ by Heeger et al.: ${ }^{16}$ paramagnetic $\rightarrow\left(T_{\mathrm{n} 1}=88.3 \mathrm{~K}\right) \rightarrow$ antiferromagnetic $\rightarrow\left(T_{\mathrm{n} 2}=81.5 \mathrm{~K}\right) \rightarrow$ weakly ferromagnetic (canted antiferromagnet). $T_{\mathrm{n} 2}$ values are essentially the same $(81,82$, and $83 \mathrm{~K})$ in almost all the published data (Table I), with the same characteristic hysteresis of 1 to $2 \mathrm{~K}$ between heating and cooling as observed in measurements of structural properties (e.g., Refs. 12, 16, 21,22, 25, 28, 29, and 33). There seems to be no doubt that this is coincident with the III $\rightarrow$ IV transition $\left(T_{\mathrm{n} 2}=T_{\mathrm{c} 3}\right)$, and the transition temperatures from both magnetic and structural measurements are therefore listed 
simply under $T_{\mathrm{c} 3}$ in Table I. Similarly, data in the literature all have $T_{\mathrm{n} 1}=87-89 \mathrm{~K},{ }^{12,16,17,22,25,28,29}$ with the exception of $95 \pm 3 \mathrm{~K}$ reported by Ogawa ${ }^{12}$ for a fine powder. These are given as $T_{\mathrm{N}}$ in Table I. Salazar et al. ${ }^{34}$ found anomalies in thermal diffusivity at $\sim 80$ and $86.6 \mathrm{~K}$, but an adjustment of their temperature scale by less than $2 \mathrm{~K}$ would bring these into register with the other data. When magnetic and diffraction measurements have both been made on the same sample, $T_{\mathrm{N}}$ and $T_{\mathrm{c} 2}$ are sometimes coincident and sometimes not (Table I), from which it can be concluded that the Néel point is fixed, while the structural transition point can vary. Sample preparation may have a significant influence on the structural transition given the difference, for example, between crystals grown by the Bridgman and Czochralski methods. ${ }^{38}$

Further anomalies in magnetic properties occur at lower temperatures but have not received such detailed attention. There is a rounded peak in both the real $\left(\chi^{\prime}\right)$ and imaginary parts $\left(\chi^{\prime \prime}\right)$ of the ac susceptibility at $\sim 65 \mathrm{~K}$ when measured at $332 \mathrm{~Hz},{ }^{28}$ and a similar peak in $\chi^{\prime}$ has also been observed at $\sim 52 \mathrm{~K}$ measured at $10 \mathrm{MHz}{ }^{48}$ Saiki and Yoshioka ${ }^{49}$ related changes in properties below $50 \mathrm{~K}$ to a spin-reorientation transition. In $\mathrm{Li}^{-}$and $\mathrm{Na}$-doped samples, changes in the magnetic properties were also attributed to a change in spin orientation below a transition temperature of $70 \mathrm{~K} .{ }^{50} \mathrm{At} \sim 12 \mathrm{~K}$ and $332 \mathrm{~Hz}$, there is a Debye-like peak in $\chi^{\prime \prime}$ and an inflection in $\chi^{\prime}{ }^{28}$ which looks like a freezing process, presumably of defects or magnetic twin walls. No evidence has yet emerged of structural changes associated with these anomalies.

In most multiferroic materials, ferroelastic/ferroelectric/ ferromagnetic transitions occur at different transition temperatures. ${ }^{51-53}$ Although these materials are classified as "multiferroic," there are no multiferroic phase transitionsonly a series of ferroic transitions. If the structural and magnetic transitions were coincident in $\mathrm{KMnF}_{3}$, or if the coupling was sufficiently strong, they would genuinely be multiferroic phase transitions, where magnetism and ferroelasticity are generated simultaneously. Essential issues are the nature and strength of magnetoelastic coupling, and these can be addressed most directly by measuring the elastic and magnetic properties of a single sample. There is one previous study of this type, but only limited data for the elastic behavior were reported. ${ }^{22}$ Elastic properties of a single crystal of $\mathrm{KMnF}_{3}$ have, therefore, been investigated by resonant ultrasound spectroscopy (RUS) in the temperature interval 11-290 K, and magnetic properties have been characterized in a SQUID magnetometer through the temperature interval $20-250 \mathrm{~K}$. The crystal came from the same original sample from which the crystal used by Gibaud et al. ${ }^{1}$ had been cut so that the elasticity and magnetic data can be correlated with results from x-ray diffraction.

Another important issue for multiferroic materials is switching, and this is controlled essentially by the dynamics of transformation-related microstructures. A particular advantage of the RUS method in this context is that it allows simultaneous determination of elastic and anelastic properties through measurement of the resonance frequencies, $f$, of small samples and the widths, $\Delta f$, of the resonance peaks. Elastic constants scale with $f^{2}$ and acoustic dissipation is expressed in terms of the inverse mechanical quality factor, $Q^{-1}=$ $\Delta f / f .{ }^{54}$ Recent experience of a number of perovskite systems

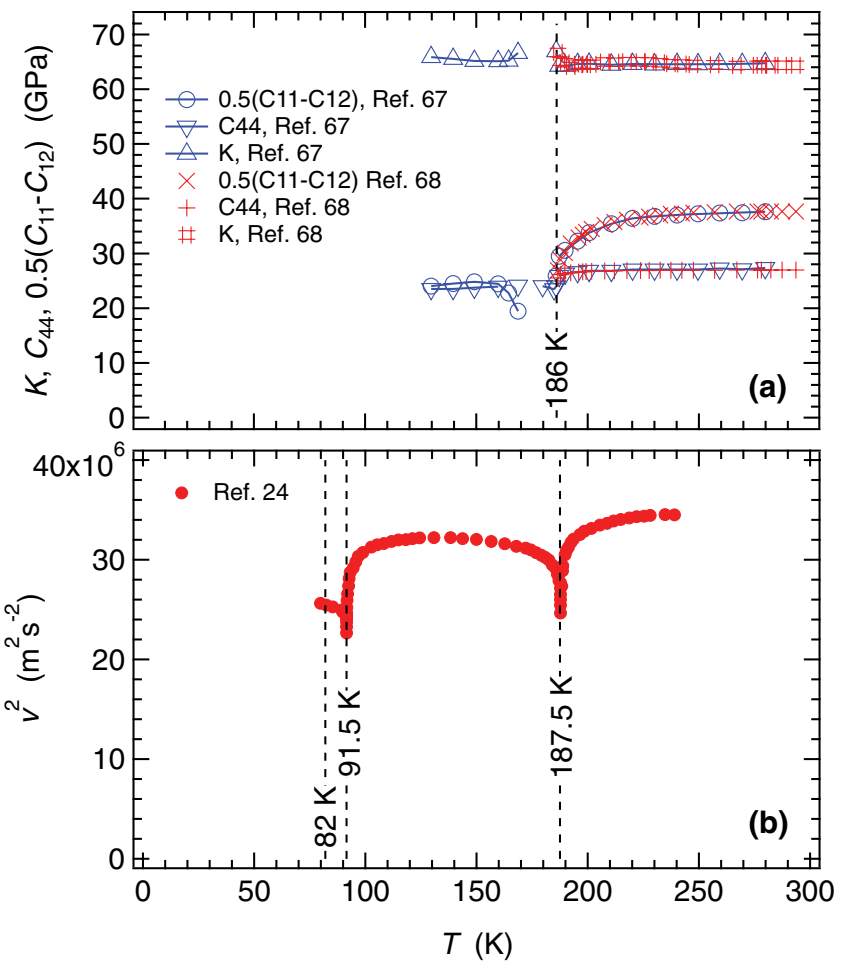

FIG. 1. (Color online) Single-crystal elastic constants for $\mathrm{KMnF}_{3}$ from the literature. (a) The original temperature scale of Reshchikova et al. (Ref. 67) has been adjusted so that the discontinuity in $C_{44}$ occurs at $186 \mathrm{~K}$ instead of $200 \mathrm{~K}$, and the temperature scale of Cao and Barsch (Ref. 68) was lowered by $3 \mathrm{~K}$ so that the two sets of data overlap. The cubic $\leftrightarrow$ tetragonal phase transition is marked by softening of $\frac{1}{2}\left(C_{11}-C_{12}\right)$ as $T \rightarrow T_{\mathrm{cl}}$ from above and below, and a small discontinuity in $C_{44}$ at $T_{\mathrm{c} 1}$. Any change in bulk modulus, $K$, is within the scatter of the data. (b) Variation in the square of the velocity of longitudinal acoustic waves parallel to [100] (proportional to $C_{11}$ ) measured at $11.7 \mathrm{MHz}$. I $\rightarrow$ II and II $\rightarrow$ III transitions at 187.5 and $91.5 \mathrm{~K}$ are marked by softening from both sides and a net softening of phase II with respect to phase I and of phase III with respect to phase II.

is that the $\sim 0.1-$ to $1-\mathrm{MHz}$ frequency range and low-stress conditions of RUS provide a particularly sensitive window into the mobility of ferroelastic twin walls. ${ }^{55-63}$ The second objective of the present study was, therefore, to investigate at RUS frequencies the remarkable mobility of domain boundary movement that has been observed at low frequencies $(\sim 0.1-$ $50 \mathrm{~Hz}$ ) in the tetragonal phase (phase II) of both pure and Ca-doped samples. ${ }^{35,37,64,65}$

\section{ELASTIC AND ANELASTIC PROPERTIES OF $\mathrm{KMnF}_{3}$}

Elastic softening at the cubic $\rightarrow$ tetragonal transition is best understood in terms of symmetry-adapted forms of the single-crystal elastic constants, $C_{11}, C_{12}$, and $C_{44}$. Eigenvalues of the elastic constant matrix are $\frac{1}{3}\left(C_{11}+2 C_{12}\right), \frac{1}{2}\left(C_{11}-C_{12}\right)$, and $C_{44}$, corresponding with irreducible representations $\Gamma_{1}^{+}$, $\Gamma_{3}^{+}$, and $\Gamma_{5}^{+}$of space group $P m \overline{3} m$ (using the notation of the space group program ISOTROPY ${ }^{66}$ ). Pulse-echo ultrasonic data of Reshchikova et al. ${ }^{67}$ collected at $30 \mathrm{MHz}$ and Cao and Barsch $^{68}$ collected at $20 \mathrm{MHz}$ are reproduced in this form in 
Fig. 1(a), with shifts in the temperature scales to place the transition temperature at $186 \mathrm{~K}$. There is a slight anomaly in the bulk modulus, $\frac{1}{3}\left(C_{11}+2 C_{12}\right)$, near the transition point, but there is no significant softening in the cubic phase ahead of the transition point. $C_{44}$ shows a very slight precursor, fluctuationinduced softening, but the main effect ahead of $T_{\mathrm{c} 1}$ is seen in $\frac{1}{2}\left(C_{11}-C_{12}\right)$ [Fig. 1(a)]. This has been analyzed by Salje and Zhang ${ }^{37}$ using a power law of form $\Delta C \propto\left(\left(T-T_{\mathrm{o}}\right) / T_{\mathrm{o}}\right)^{-\kappa}$, where $\Delta C$ is the amount of softening with respect to values of the elastic constants extrapolated from high temperatures and $T_{\mathrm{o}}$ is the critical temperature for the phase transition. Values of $\kappa$ produced from fits to the data vary between $\sim 0.4$ and 1.0 for data collected at both low $(\sim 10-\mathrm{Hz})$ and high $(20-\mathrm{MHz})$ frequencies.

Softening below $T_{\mathrm{cl}}$ occurs by relaxations of the order parameter due to coupling with strain. From the data of Reshchikova et al. ${ }^{67}$ in Fig. 1(a), the change in bulk modulus across the transition is less than the experimental uncertainty of extrapolation from the stability field of the cubic phase, $C_{44}$ softens by $\sim 11 \%$ and $\frac{1}{2}\left(C_{11}-C_{12}\right)$ by $\sim 36 \%$, as averaged over all twin orientations present in the sample. Similar softening is seen in pulse-echo ultrasonic data for $C_{11}$ at $5 \mathrm{MHz}^{20}$ and for longitudinal waves traveling parallel to [111] at $15 \mathrm{MHz}{ }^{27}$ The form of this softening can be accounted for using the model of Slonczewski and Thomas, ${ }^{35,69}$ in the same manner as has been used to account quantitatively for softening below the same cubic $\rightarrow$ tetragonal tilting transition in $\mathrm{SrTiO}_{3}{ }^{70}$ Because the transition in $\mathrm{KMnF}_{3}$ is close to tricritical, there is a marked nonlinear softening as the transition point is approached from below ${ }^{35}$ (see also, Ref. 71).

Successive tilting transitions in $\mathrm{Sr}(\mathrm{Ti}, \mathrm{Zr}) \mathrm{O}_{3}$ give a characteristic sequence of minima in the shear modulus, ${ }^{55,56}$ and the same pattern would be expected for a sequence of similar transitions in $\mathrm{KMnF}_{3}$. This is seen in data for $C_{11}$ and $\frac{1}{2}\left(C_{11}+C_{13}\right)+C_{55}$ from Ca-doped samples, ${ }^{35}$ with minima at temperatures close to $T_{\mathrm{c} 1}, T_{\mathrm{c} 2}$, and $T_{\mathrm{c} 3}$ from Gibaud et al. ${ }^{1}$ $C_{44}$ shows minima at $T_{\mathrm{c} 1}$ and $T_{\mathrm{c} 3}$ but only a break in slope at $T_{\mathrm{c} 2} .{ }^{35}$ The only data for pure $\mathrm{KMnF}_{3}$ are acoustic velocities given by Fossheim et al. ${ }^{24}$ for longitudinal waves parallel to [100], i.e., relating to $C_{11}$, measured at $11.7 \mathrm{MHz}$. These are reproduced here in Fig. 1(b) and show minima at $187.5 \mathrm{~K}$ $\left(T_{\mathrm{c} 1}\right)$ and $91.5 \mathrm{~K}\left(T_{\mathrm{c} 2}\right)$. They do not extend to low enough temperatures to characterize any softening at $T_{\mathrm{c} 3}$, however.

Data collected in the frequency range $0.2-32 \mathrm{~Hz}$ by dynamical mechanical analysis (DMA) with three-point bending geometry show that domain wall freezing occurs near $160 \mathrm{~K}$ and is determined by pinning to defects with a fairly narrow range of activation energies near $0.43 \mathrm{eV}^{37}$ Data for a Cadoped sample $\left(\mathrm{KMn}_{0.997} \mathrm{Ca}_{0.003} \mathrm{~F}_{3}\right)$ could be fit better with a Vogel-Fulcher description of the freezing and an activation energy of $0.23 \mathrm{eV} .{ }^{37}$ Additional domain freezing temperatures, near $105 \mathrm{~K}$, have been found for a thicker Ca-doped crystal with the same composition in parallel plate geometry. ${ }^{35,64,65}$ Analysis in terms of glassy behavior gave an activation energy of $0.14 \mathrm{eV}^{35}$ Significant attenuation has also been reported between $T_{\mathrm{c} 1}$ and $T_{\mathrm{c} 2}$ in pulse-echo ultrasonic measurements at 10 and $11.7 \mathrm{MHz}^{22,24}$ On the other hand, there appears to be no influence of twin-wall mobility on elastic properties measured by the plane-wave resonance method at $15 \mathrm{MHz}{ }^{35}$ The low-frequency lossy behavior disappears abruptly below the transition temperature to phase IV in Ca-doped samples, ${ }^{35}$ and the same abrupt change has been observed in pure $\mathrm{KMnF}_{3}$ at $10 \mathrm{MHz}{ }^{22}$ This coincides with the more general view that ferroelastic twins are effectively immobile in perovskites with the Pnma structure, ${ }^{37,55,56,72-75}$ with the possible exception of $\mathrm{BaCeO}_{3} .{ }^{62}$ Here we report freezing behavior of twin walls at $\sim 135 \mathrm{~K}$ but propose that the loss mechanism at $\sim 1 \mathrm{MHz}$ is not quite the same as occurs at $\sim 10 \mathrm{~Hz}$. We have also observed significant anelastic losses in phase III, which diminish abruptly in phase IV. If there is any significant coupling between magnetic order parameters and strain, it is inevitable that magnetic twin walls might move in response to an applied stress and contribute to the anelastic loss.

Acoustic attenuation at higher frequencies $(\sim 10-600 \mathrm{MHz})$ also reveals details of relaxation processes associated with the I $\rightarrow$ II transition. ${ }^{18,24,27,67,76}$ These are believed to relate to the dynamics of ordered clusters just above $T_{\mathrm{c} 1}$, with some sensitivity to impurities, and Landau-Khalatnikov damping below $T_{\mathrm{c} 1}$, with relaxation times related to the order parameter dynamics, rather than to relaxation properties of the transformation microstructure. ${ }^{27,76}$

\section{EXPERIMENTAL METHODS}

The sample used in the present study for elasticity and magnetic measurements was a pale pink single crystal from the same source of Bridgman-grown crystals of $\mathrm{KMnF}_{3}$ as used by Gibaud et al. ${ }^{1}$ for x-ray diffraction experiments. It was in the form of a slice, $1.068 \mathrm{~mm}$ thick, with faces parallel to (001) and polished on one side. It had an irregular shape in the second and third dimensions (very approximately $10 \mathrm{~mm}$ across) and mass $0.3354 \mathrm{~g}$. The presence of a secondary phase with optical birefringence was detected in a polarizing light microscope. It was present as a dust of very small crystallites generally distributed throughout the sample but also with a narrow band containing a higher concentration of crystallites. The most likely impurity phase, $\mathrm{MnF}_{2}$, is tetragonal at room temperature and undergoes an antiferromagnetic transition at $68 \mathrm{~K}$ that is known to give rise to small changes in elastic properties (e.g., Ref. 77). Parts of the original sample of Gibaud et al. ${ }^{1}$ were used also by Hayward et al. ${ }^{11}$ for measurements of heat capacity and twin angles, by Romero et al. ${ }^{8,9}$ and Gallardo et al. ${ }^{10}$ for heat capacity measurements, by Schranz et al. ${ }^{35,64}$ and Salje and Zhang ${ }^{37}$ for low-frequency elasticity measurements, by Schranz et al ${ }^{35}$ for elasticity measurements at high frequencies, and by Guennou et al. ${ }^{78}$ for single-crystal diffraction measurements at high pressures.

RUS measurements were made in an Orange helium flow cryostat with dynamic resonance system (DRS) Modulus II electronics, as described by McKnight et al. ${ }^{79}$ Temperature was controlled by a Lakeshore 340 controller, and the temperature at the sample measured with a DT670 silicon diode. Accuracy of the recorded temperatures is believed to be better than $\pm 1 \mathrm{~K}$, and the stability of the system is at least $\pm 0.1 \mathrm{~K}$. The sample was held lightly across its large faces directly between the piezoelectric (PZT) transducers. Automated data collection involved cooling in $30-\mathrm{K}$ steps from 280 to $10 \mathrm{~K}$, with a period of $15 \mathrm{~min}$ allowed for thermal equilibration. The heating sequence was then 10 $60 \mathrm{~K}$ in $5-\mathrm{K}$ steps $(+15-$ min equilibration time at each 
temperature), 60-100 in 1-K steps (+10-min equilibration), $100-170 \mathrm{~K}$ in $5-\mathrm{K}$ steps (+15-min equilibration), 170-200 in $1-\mathrm{K}$ steps (+10-min equilibration), and 200-295 in 5-K steps (+15-min equilibration). Spectra were transferred to the software package IGOR PRO (Wavemetrics) for analysis, and an asymmetric Lorentzian function was used to fit selected resonance peaks in order to extract values of $f$ and $\Delta f$ for determinations of $f^{2}$ and $Q^{-1}$ as a function of temperature.

Magnetic measurements were made in a MPMS XL SQUID magnetometer. Many details of the measuring system are given by McElfrish et al. ${ }^{80}$ In the first experiment, the $\mathrm{KMnF}_{3}$ crystal was held in the magnetometer in an arbitrary orientation, and the magnetic moment measured with the magnetic field switched off. Outputs from the system were temperature, $T$, and magnetic moment in electromagnetic moment units (emu). Data were collected in a heating and cooling sequence as follows:

Sample cooled to $20 \mathrm{~K}$.

Sweep: $1 \mathrm{~K} . \mathrm{min}^{-1}$ from 20 to $70 \mathrm{~K}$ with data collection every $0.5 \mathrm{~K}$.

Settle: 70.2 to $100 \mathrm{~K}$ with settling and reading at $0.2-\mathrm{K}$ intervals.

Sweep: $1 \mathrm{~K} \cdot \mathrm{min}^{-1}$ from 100 to $250 \mathrm{~K}$ with data collection every $1 \mathrm{~K}$.

Sweep: $1 \mathrm{~K} \cdot \mathrm{min}^{-1}$ from 250 to $100 \mathrm{~K}$ with data collection every $1 \mathrm{~K}$.

Settle: 99.9 to $70.1 \mathrm{~K}$ with settling and reading at $0.2-\mathrm{K}$ intervals.

Cool to $20 \mathrm{~K}$, followed by sweep 20 to $45 \mathrm{~K}$, with data collection every $0.5 \mathrm{~K}$.

"Sweep" means data collection during continuous heating/ cooling, while "settle" means equilibration at each temperature before data collection.

When the crystal was removed from the magnetometer, it was accidentally broken into three pieces. One of these, weighing $0.1057 \mathrm{gm}$, was returned to the magnetometer and used to test for magnetic hysteresis at 40, 60, 85, 120, and $220 \mathrm{~K}$ during both cooling and warming. A field of 50 Oe was applied during cooling, and heating and fields up to $+15 \mathrm{kOe}$ were applied at the fixed temperatures.

\section{RESULTS}

\section{A. Magnetism}

Variations of the moment and (moment $)^{-1}$, which is proportional to the inverse susceptibility, in a nominally zero field are shown in Fig. 2. The overall pattern is shown in Fig. 2(a), while Figs. 2(b) and 2(c) show details of the evolution in the intervals $50-110 \mathrm{~K}$ and $150-240 \mathrm{~K}$, respectively. The presence of small but measurable moments for the paramagnetic tetragonal phase (phase II) implies that there must have been some residual magnetic field in the instrument, and these reveal significant details of the magnetic behavior through the entire temperature interval over which data were collected. The largest moment was observed in the sample at $20 \mathrm{~K}$ after cooling directly from room temperature. This reduced steeply on heating to $\sim 60 \mathrm{~K}$, where there is a marked break in slope [Fig. 2(a)]. There is a sharp discontinuity in (moment) ${ }^{-1}$ at $82.2 \mathrm{~K}$ and a smaller but equally distinct discontinuity at $87.3 \mathrm{~K}$ [Fig. 2(b)].

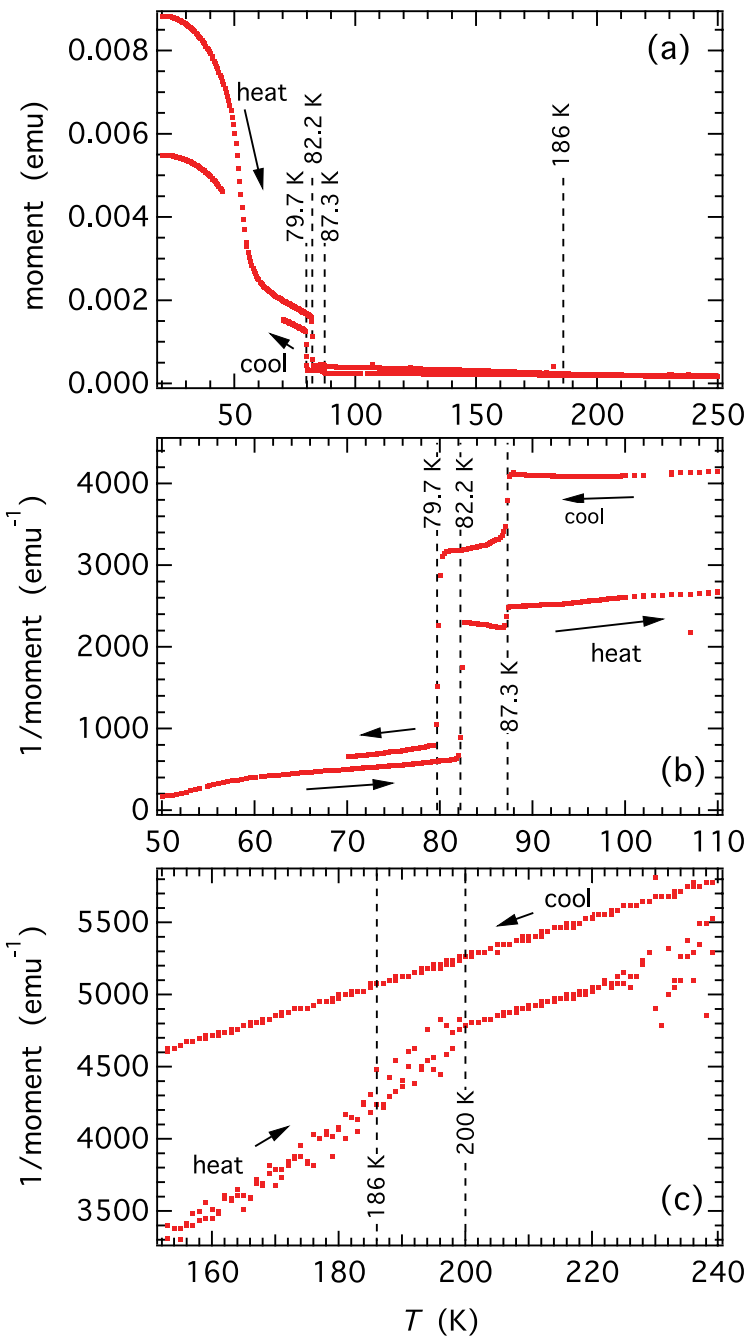

FIG. 2. (Color online) SQUID measurements of the magnetic moment in $\mathrm{KMnF}_{3}$ under conditions of nominally zero applied field. (a) The phase transitions near $T_{\mathrm{c} 2}=87.3 \mathrm{~K}$ and $T_{\mathrm{c} 3}=80 / 82 \mathrm{~K}$ are clearly visible. (b) Detail from (a) plotted as (magnetic moment $)^{-1}$, proportional to inverse susceptibility, showing abrupt discontinuities at 87.3 and $79.7 \mathrm{~K}$ during cooling or at 82.2 and $87.3 \mathrm{~K}$ during heating. (c) Detail from (a) plotted as (magnetic moment) $)^{-1}$, showing a subtle hysteresis in magnetic properties through the cubic $\leftrightarrow$ tetragonal transition. Both phases are paramagnetic, so the variations are assumed to be due to paramagnetic anisotropy and differences in the topology of transformation twins present in the tetragonal phase. The break in slope at $200 \mathrm{~K}$ during heating suggests that some microstructure, probably tweed, remains in the cubic phase above $T_{\mathrm{c} 1}=186 \mathrm{~K}$.

A slight break in slope then occurs near $200 \mathrm{~K}$ [Fig. 2(c)]. On subsequent cooling, the first feature is the small discontinuity at $87.3 \mathrm{~K}$, followed by a steeper discontinuity at $79.7 \mathrm{~K}$. At the lowest temperatures the pattern of evolution was the same as in the initial heating sequence, although the absolute values were smaller. Linear fits to the heating data put the break in slope during the heating sequence at $\sim 200 \mathrm{~K}$ [Fig. 2(c)].

Figure 3(a) shows the effect of varying magnetic field strength on the measured values of the moment at selected fixed temperatures, with details of the hysteresis behavior as a function of applied field, $H$, shown in Fig. 3(b). Ferromagnetic 

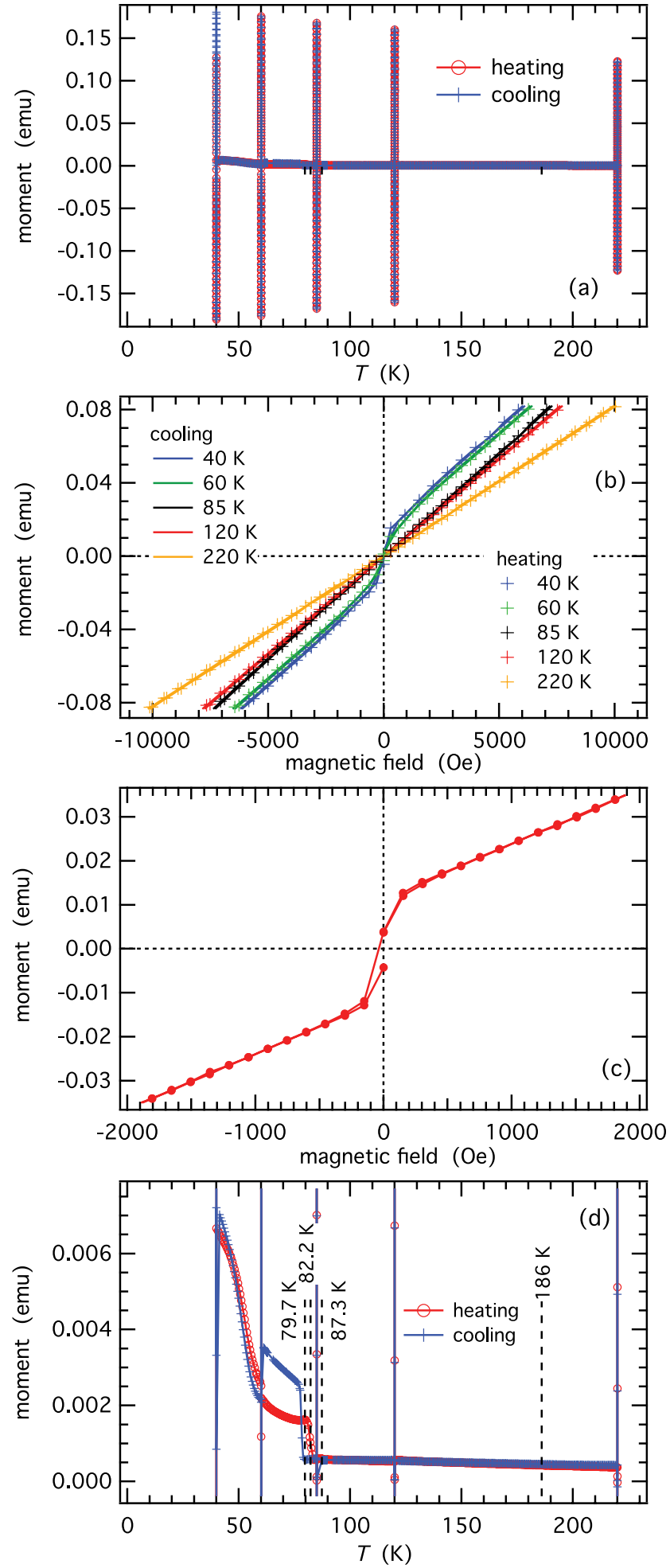

FIG. 3. (Color online) SQUID measurements of the magnetic moment in $\mathrm{KMnF}_{3}$ under conditions of applied field. (a) Moment at fields up to $\pm 15 \mathrm{kOe}$ for specific temperatures and a field of $50 \mathrm{Oe}$ during heating and cooling between these temperatures. (b) Detail of hysteresis measurements showing that the crystal is paramagnetic at 220,120 , and $85 \mathrm{~K}$ but has a ferromagnetic moment at 60 and $40 \mathrm{~K}$. (c) Detail of the hysteresis measurements made at $40 \mathrm{~K}$. (d) Detail of moment measurements as a function of temperature, showing significant differences before and after application of the $15-\mathrm{kOe}$ field in the stability field of phase IV. hysteresis loops were observed at 40 and $60 \mathrm{~K}$, in the stability field of phase IV, but the moment vs field plots are linear through the origin at $85 \mathrm{~K}$ (phase III) and 120 and $220 \mathrm{~K}$ (phase II). Details of the hysteresis loop measured at $40 \mathrm{~K}$ are shown in Fig. 3(c). The results obtained at these fixed temperatures were the same when measured during cooling and heating. A feature of the hysteresis behavior in the stability field of phase IV was that application of the strong field caused a substantial change in moment when measured again at 50 Oe [Fig. 3(d)], implying that a change in the configuration of magnetic domains had been induced.

If the impurity phase observed optically is $\mathrm{MnF}_{2}$, some anomaly in magnetic moment would have been expected in the vicinity of the Néel point, $68 \mathrm{~K}$, as shown, for example, by Schleck et al. ${ }^{81}$ That this is not observed in any of the SQUID data implies either that the tenuous identification of the birefringent particles is incorrect or that they are present in a volume proportion which is too small to influence the bulk magnetic properties of the whole $\mathrm{KMnF}_{3}$ sample.

\section{B. Elastic and anelastic behavior}

Segments of the resonance spectra collected during heating through the phase transitions are shown as a stack in Fig. 4. The $y$ axis is amplitude, but the spectra have been shifted in proportion to the temperature at which they were collected. With increasing temperature up to $\sim 83 \mathrm{~K}$, relatively sharp peaks all soften (reduce in frequency). The softening trend reverses abruptly and resonance peaks above $83 \mathrm{~K}$ are clearly broadened. This broadening increases with further increasing temperature until the peaks are not visible at the scale shown. Broad peaks then reappear and soften towards $\sim 185 \mathrm{~K}$, where they reach a minimum frequency. Above $\sim 185 \mathrm{~K}$, the peaks are narrower and stiffen with increasing temperature. This overall pattern is followed for all the resonance peaks observed, although fewer peaks were seen at higher frequencies in the temperature range $\sim 83-185 \mathrm{~K}$ due to the strong attenuation.

Variations of $f^{2}$ and $Q^{-1}$ for selected peaks give a quantitative picture of the qualitative pattern shown by the primary spectra (Fig. 5). In order to show results for different peaks on the same graph, values of $f^{2}$ have been scaled so as to overlap near room temperature. The two peaks shown between 83 and $185 \mathrm{~K}$ could be matched with peaks above $185 \mathrm{~K}$ with some confidence but could not be followed across the discontinuity at $83 \mathrm{~K}$. They do not provide a constrained measure of the amount of softening (or stiffening) of elastic constants across the III $\leftrightarrow$ IV transition, therefore. The frequencies of several peaks in this low-temperature interval have been scaled to overlap at $10 \mathrm{~K}$. The I $\leftrightarrow$ II transition is marked by a minimum in $f^{2}$, comparable to the steep minimum seen in different elastic constants at higher frequencies ${ }^{20,24,27,35}$ [Fig. 1(b)], and a steep increase in $Q^{-1}$ in the stability field of phase II. In the stability field of the cubic structure, $Q^{-1}$ values for a few peaks are markedly higher than for others and show a steep increase above $\sim 250 \mathrm{~K}$. The stability field of phase II is marked by the combination of a peak in $Q^{-1}$ near $135 \mathrm{~K}$ and a change in $f^{2}$ values, which has the typical form of Debye-like loss behavior due to some freezing process. There are no gross features in the RUS data at $87 \mathrm{~K}$ that might correspond with the change in magnetic susceptibility, but there is a very slight 


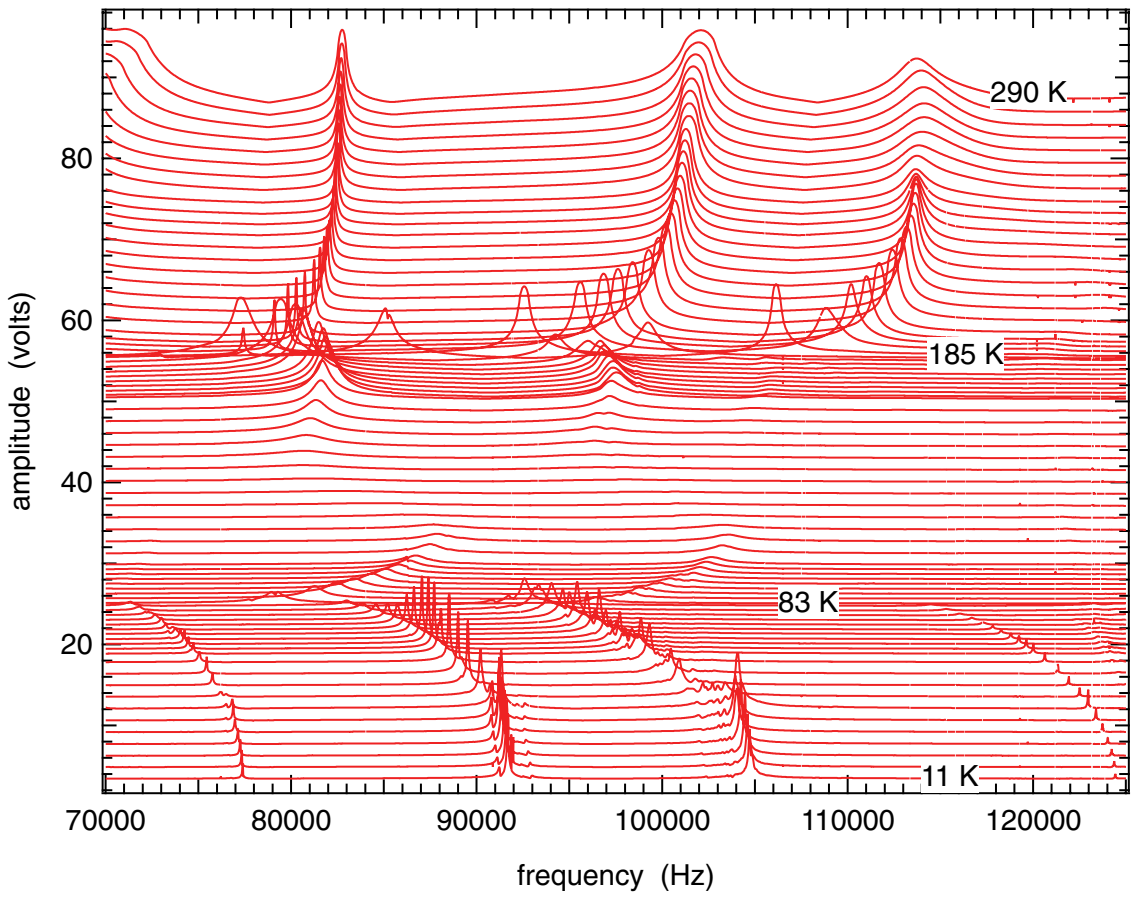

FIG. 4. (Color online) Segments of RUS spectra collected during heating, stacked in proportion to the temperature at which they were collected. There are obvious minima in peak frequencies at $\sim 185$ and $83 \mathrm{~K}$ but no obvious change at $87 \mathrm{~K}$. Marked broadening of resonance peaks in the temperature interval $\sim 83-185 \mathrm{~K}$ is attributed to the mobility of transformation twin walls. change in slope of $f^{2}$ with temperature, as shown for one of the resonance peaks in Fig. 6. There is a minimum in $f^{2}$ and a steep change in $Q^{-1}$ at $83 \mathrm{~K}$, however. In the stability field of phase IV, $Q^{-1}$ drops off markedly to values that are lower than those of the sharpest peaks in spectra from the cubic structure. There are no further anomalies in either $f^{2}$ or $Q^{-1}$ below $83 \mathrm{~K}$, which might indicate the effects of any further structural or magnetic transitions. There is also no evidence for an elastic anomaly at $68 \mathrm{~K}$, which would be indicative of the antiferromagnetic ordering transition in $\mathrm{MnF}_{2} \cdot{ }^{77}$ The

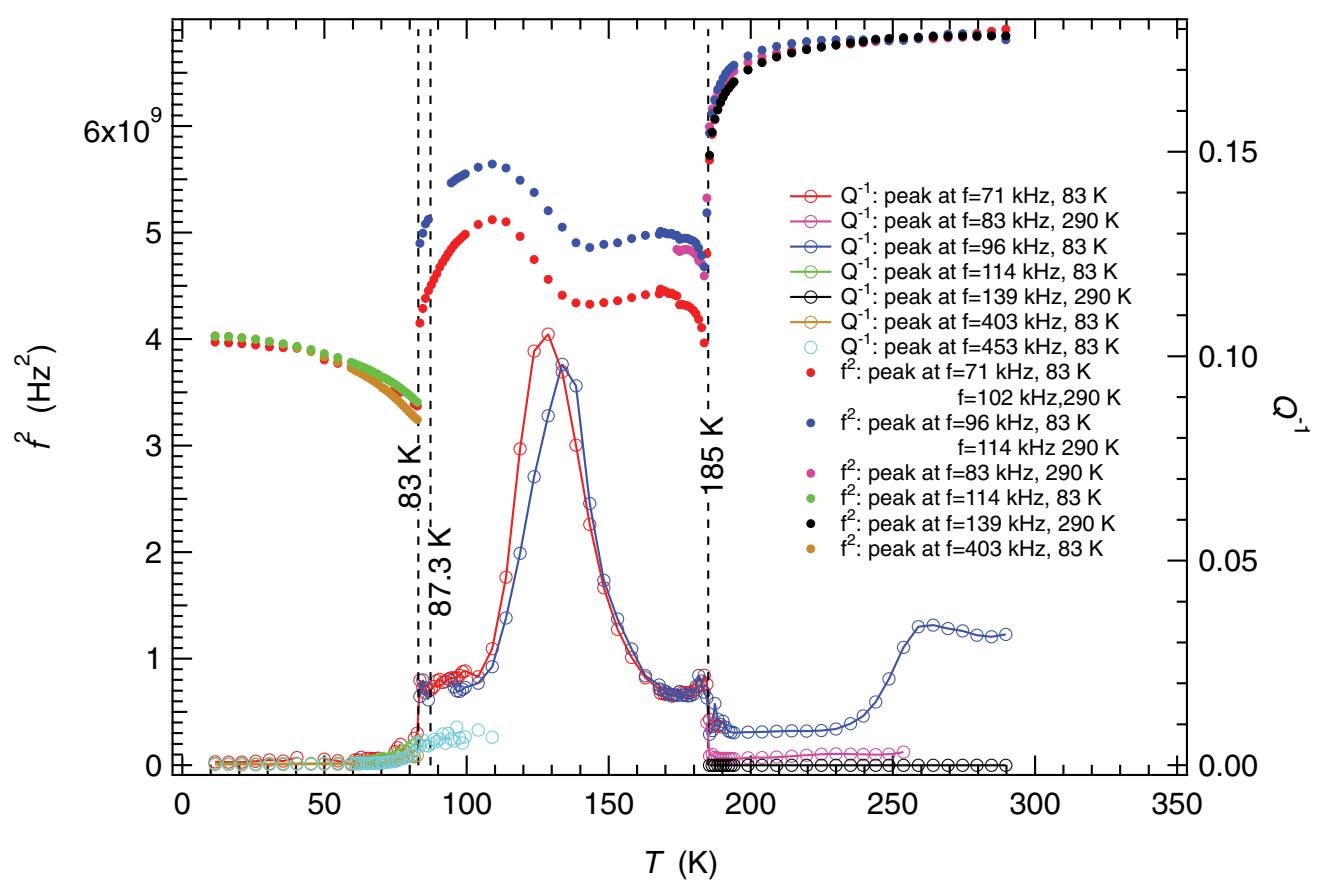

FIG. 5. (Color online) Variations of the square of the frequency, $f$, and inverse mechanical quality factor, $Q^{-1}$, for selected peaks in RUS spectra collected during heating. Values of $f^{2}$ for different peaks have been rescaled so as to overlap near room temperature. The step at $185 \mathrm{~K}$ is correct in magnitude in the sense that it was possible to follow peaks through the transition at this temperature. The magnitude of the step at $83 \mathrm{~K}$ is not reliable, however, since it was not possible to follow peaks through the transition at this temperature with any degree of confidence. The $f^{2}$ values for different peaks below $83 \mathrm{~K}$ were rescaled to produce an overlap near $10 \mathrm{~K}$. The peak in $Q^{-1}$ near $130 \mathrm{~K}$ and the increase in $f^{2}$ below this temperature are typical of a Debye-like dissipation process and are attributed here to freezing of transformation twin-wall motion by pinning to defects. 


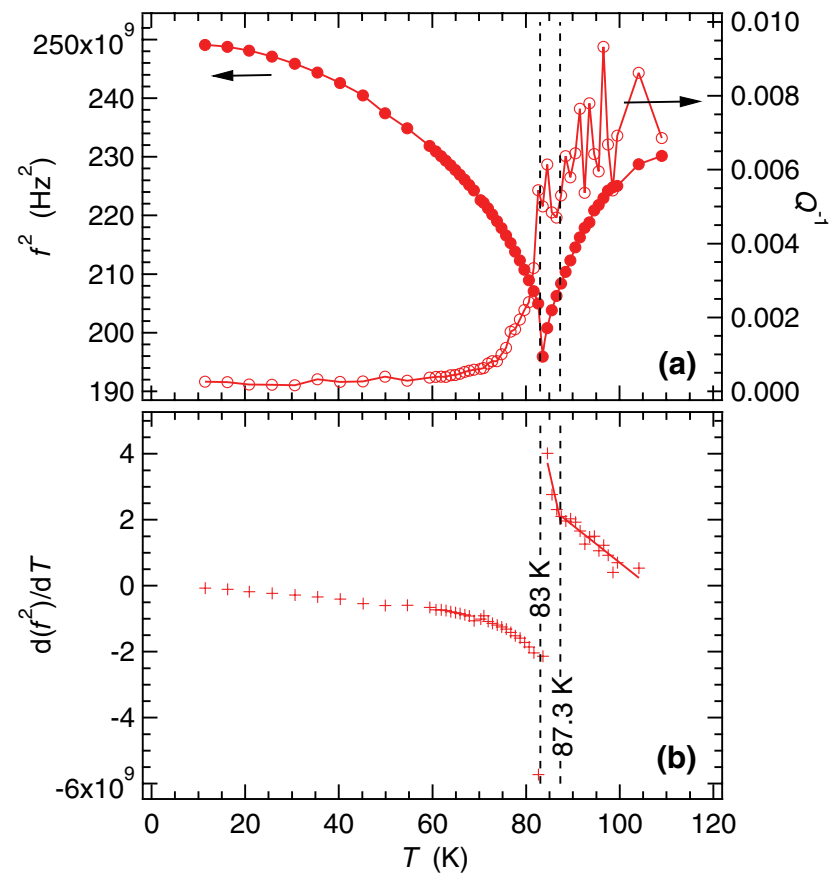

FIG. 6. (Color online) (a) Details of the variations with temperature of $f^{2}$ and $Q^{-1}$ for a resonance peak with $f \approx 453 \mathrm{kHz}$ at $83 \mathrm{~K}$ (data collected during heating). There is clearly a change in the trend of both $f^{2}$ and $Q^{-1}$ at $83 \mathrm{~K}$ but no obvious change at $87 \mathrm{~K}$. (b) There is a clear break in slope of $f^{2}$ as a function of temperature at $87 \mathrm{~K}$; straight lines are fits to the data in two different temperature intervals.

only possible correlation between results from the present magnetic and elasticity measurements is that the steep increase in susceptibility below $\sim 60 \mathrm{~K}$ [Fig. 2(a)] coincides with the temperature below which $Q^{-1}$ values become constant and very low [Fig. 6(a)].

Some of the lowest-frequency resonance modes of a small rectangular parallelepiped cut for a cubic single crystal can be "pure" shear modes in the sense that they are determined essentially by $C_{44}$ or by $\frac{1}{2}\left(C_{11}-C_{12}\right)$ alone. The resonance modes of a thin plate are more likely to resemble the types of motion that would occur in a three-point bending measurement. The elastic constants of these will tend to be of the form of Young's modulus for directions within the plane of the plate, such as $Y[100]$ and $Y$ [110]. Explicit expressions for these have been given by Carpenter et $\mathrm{l}^{58}{ }^{58}$ (following Nye ${ }^{82}$ )

$$
\begin{aligned}
& Y_{[110]}=\left(\frac{C_{11}}{2\left(C_{11}-C_{12}\right)\left(C_{11}+2 C_{12}\right)}+\frac{1}{4 C_{44}}\right)^{-1} \\
& Y_{[100]}=\left(\frac{C_{11}+C_{12}}{\left(C_{11}-C_{12}\right)\left(C_{11}+2 C_{12}\right)}\right)^{-1},
\end{aligned}
$$

and the single-crystal elastic constants of Cao and Barsch ${ }^{68}$ have been used to calculate their variations with temperature for comparison with $f^{2}$ data for resonance peaks (Fig. 7). The form of softening for all the observed resonance modes is described correctly and is consistent with the expectation that all such bending modes depend to some extent on $\frac{1}{2}\left(C_{11}-C_{12}\right)$.

Following Carpenter et al. ${ }^{60}$ the Debye peaks at $\sim 130 \mathrm{~K}$ would be expected to depend on an activation energy, $E_{\mathrm{a}}$,

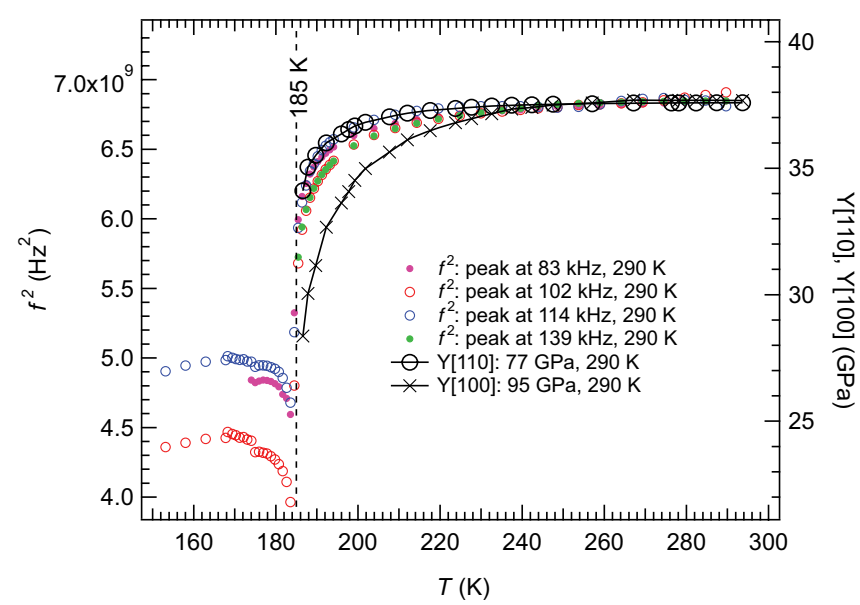

FIG. 7. (Color online) Comparison of $f^{2}$ data (left axis) for selected resonance peaks with values of Young's modulus for [110] and [100] directions calculated Eqs. (1) and (2) using the single-crystal elastic constants of $\mathrm{CaO}$ and Barsch (Ref. 68; note that the temperature scale of $\mathrm{Cao}$ and Barsch was lowered by $3 \mathrm{~K}$, as shown in Fig. 1). Young's modulus values (right axis) have been rescaled so as to match the RUS data near room temperature. The observed resonance frequencies are consistent with resonances of the single-crystal plate being influenced by motions comparable with three point bending, i.e., including the influence of both $\frac{1}{2}\left(C_{11}-C_{12}\right)$ and $C_{44}$.

and the inverse of an attempt frequency, $\tau_{0}$, for a thermally activated process according to

$$
\tau=\tau_{\mathrm{o}} \exp \left(\frac{E_{\mathrm{a}}}{\mathrm{R} T}\right),
$$

where $\tau$ is the relaxation time at temperature $T$. The Debye peak itself is expected to depend on the angular frequency, $\omega$ $(=2 \pi f)$, of an applied stress according to

$$
Q^{-1}=\Delta \frac{\omega \tau}{1+\omega^{2} \tau^{2}} .
$$

In the case of a standard linear solid

$$
\Delta=\frac{C_{\mathrm{U}}-C_{\mathrm{R}}}{C_{\mathrm{R}}} \quad\left(\text { for }\left(C_{\mathrm{U}}-C_{\mathrm{R}}\right) \ll C_{\mathrm{R}}\right),
$$

where $C_{\mathrm{U}}$ is the elastic modulus for the unrelaxed state and $C_{\mathrm{R}}$ the elastic modulus of the relaxed state. ${ }^{83}$ The maximum value of $Q^{-1}, Q_{\mathrm{m}}^{-1}$, occurs at temperature $T_{\mathrm{m}}$, and is equal to $\Delta / 2$. A single peak measured as a function of temperature at approximately constant frequency can be described by ${ }^{84,85}$

$$
Q^{-1}(T)=Q_{\mathrm{m}}^{-1}\left[\cosh \left\{\frac{E_{a}}{\operatorname{Rr} r_{2}(\beta)}\left(\frac{1}{T}-\frac{1}{T_{\mathrm{m}}}\right)\right\}\right]^{-1} .
$$

Here $r_{2}(\beta)$ is a width parameter that arises from any spread in relaxation times for the dissipation process. The width of a peak measured as a function of temperature at constant frequency is determined essentially by the value of the activation energy and the value of $r_{2}(\beta)$. Fits of (6), including linear baselines, are shown in Fig. 8, with $E_{\mathrm{a}} / r_{2}(\beta), Q_{\mathrm{m}}^{-1}$, and $T_{\mathrm{m}}$ values of $15 \mathrm{~kJ}^{\mathrm{mole}}{ }^{-1}(\sim 0.16 \mathrm{eV}), 0.087,128 \mathrm{~K}$, and $16 \mathrm{~kJ} \cdot \mathrm{mole}^{-1}(\sim 0.17 \mathrm{eV})$, and 0.081 and $132 \mathrm{~K}$, respectively, for frequencies of 82 and $98 \mathrm{kHz}$ at $T_{\mathrm{m}}$. The relaxation time at $132 \mathrm{~K}$ is $1.6 \times 10^{-6} \mathrm{~s}(\omega \tau=1)$, and using $r_{2}(\beta)=1$, 


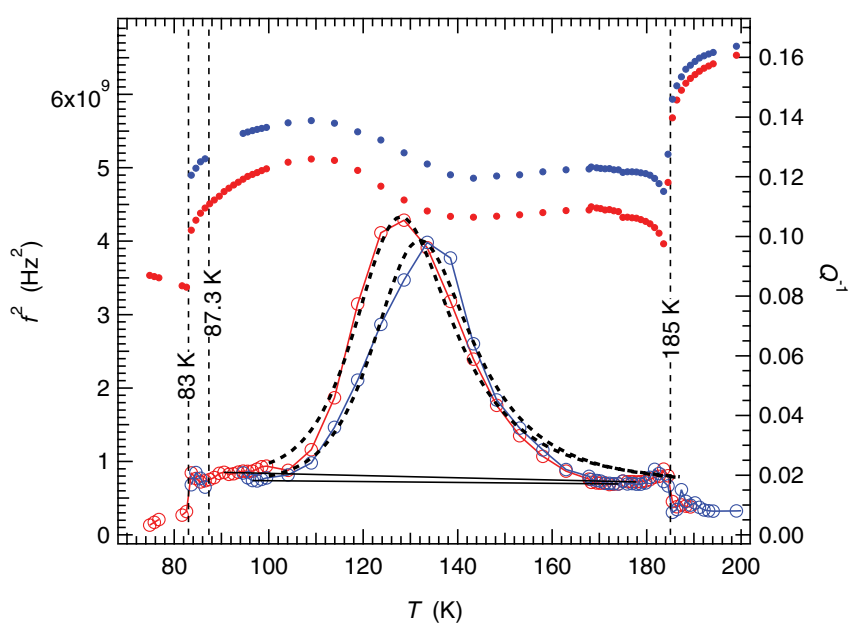

FIG. 8. (Color online) Fits of (6) to the Debye-like peaks in $Q^{-1}$ from Fig. 5. Solid lines are baselines for the fits, and broken lines are the fits themselves.

$E_{\mathrm{a}}=16 \mathrm{~kJ} . \mathrm{mole}^{-1}$ gives $\tau_{\mathrm{o}}=8 \times 10^{-13} \mathrm{~s}$. If there is a Gaussian spread of activation energies, as specified by the value of $\beta$, the value of $E_{\mathrm{a}}$ will be larger and the value of $\tau_{\mathrm{o}}$ smaller. For example, if $r_{2}(\beta)=1.25$ (for $\beta=1$ ), then $E_{\mathrm{a}}=$ $20 \mathrm{~kJ} . \mathrm{mole}{ }^{-1}$ and $\tau_{\mathrm{o}}=2 \times 10^{-14} \mathrm{~s}$.

Finally, if $Q_{\mathrm{m}}^{-1}=0.087$ (or 0.081 ), (4) gives $\Delta=17.4 \%$ (or $16.2 \%$ ). This is consistent with the changes in $f^{2}$ across the temperature interval $\sim 155-105 \mathrm{~K}$ in Fig. 5, which imply a softening of the related combinations of elastic constants by $\sim 17 \%$ (or $\sim 15 \%$ ). In other words the crystal is effectively $17 \%$ elastically softer due to the mobility of some defects on a time scale of $\sim 10^{-6} \mathrm{~s}$.

\section{DISCUSSION}

\section{A. Transition sequence}

The magnetic and elasticity data from the present study and the structural data of Gibaud et al. ${ }^{1}$ provide a clear view of the relationship between magnetic and structural phase transitions in a single sample of $\mathrm{KMnF}_{3}$. The first-order canting transition, with $\sim 2-\mathrm{K}$ hysteresis, is identical to the structural transition III $\rightarrow$ IV, while $T_{\mathrm{N}}$ and $T_{\mathrm{c} 2}$ are coincident within an experimental uncertainty of $\sim 1 \mathrm{~K}$. The value of $T_{\mathrm{c} 1}$ is also within $\sim 1 \mathrm{~K}$ of the values from heat capacity measurements $\left(186.0 \mathrm{~K}^{11}\right)$ and from elasticity measurements at $15 \mathrm{MHz}\left(186.7 \mathrm{~K}^{35}\right)$. The elasticity data of Schranz et al ${ }^{35}$ indicate $T_{\mathrm{c} 2}=91.0 \mathrm{~K}$, but it is not clear that this can necessarily be distinguished from $88 \mathrm{~K}$. Variations of $C_{11}$ and $C_{44}$ from Schranz et al. ${ }^{35}$ for a sample doped with $1.8 \% \mathrm{Ca}$ are reproduced in Fig. 9. Transition temperatures taken from the minima in $C_{11}$ are systematically $\sim 3 \mathrm{~K}$ higher than those reported for a piece of the same sample by Gibaud et al. ${ }^{1}$ Transition temperatures estimated from the positions of anomalies in $C_{44}$ are 2 to $3 \mathrm{~K}$ lower than those suggested by $C_{11}$, however, and this perhaps represents the experimental uncertainty in the absolute values of their temperature scale. Adding all this to data from the literature (Table I) confirms $T_{\mathrm{c} 1} \approx 186, T_{\mathrm{N}} \approx 88, T_{\mathrm{c} 3} \approx 83 \mathrm{~K}$ for almost all samples, however prepared. In contrast, the value of $T_{\mathrm{c} 2}$ clearly varies between samples and must be

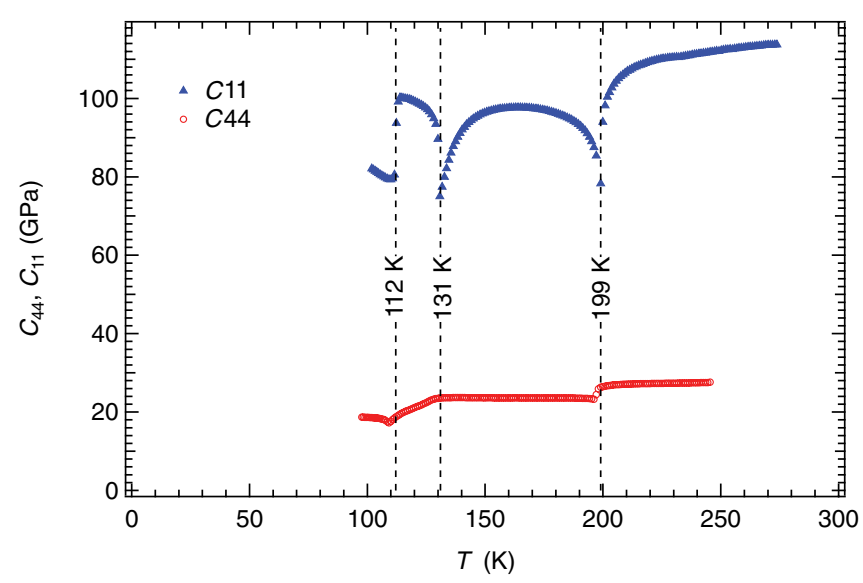

FIG. 9. (Color online) Variations of single-crystal elastic constants of $\mathrm{K}_{0.982} \mathrm{Ca}_{0.018} \mathrm{~F}_{3}$ measured at $15 \mathrm{MHz}$, from Schranz et al. ${ }^{35}$ Successive phase transitions corresponding to I $\leftrightarrow$ II $(199 \mathrm{~K})$, II $\leftrightarrow$ III $(131 \mathrm{~K})$, and III $\leftrightarrow \mathrm{IV}(112 \mathrm{~K})$ are apparent. The transition temperatures shown are taken from the data for $C_{11}$. Data for $C_{44}$ have changes in trend that appear to place the transition temperatures a few degrees lower than these values. Note that all three transitions are marked by discontinuities in $C_{11}$ but that the transition near $131 \mathrm{~K}$ gives a change in slope for $C_{44}$. This is consistent with a strain/order parameter coupling model describing a sequence $P m \overline{3} m \leftrightarrow I 4 / m c m \leftrightarrow C m c m \leftrightarrow P n m a$.

sensitive to defects, impurities, or variations in stoichiometry. Replacing $\mathrm{K}^{+}$with a smaller cation, such as $\mathrm{Li}^{+}, \mathrm{Na}^{+}$, or $\mathrm{Ca}^{2+}$, causes $T_{\mathrm{c} 1}$ and $T_{\mathrm{c} 2}$ to increase, ${ }^{1,50,86}$ and even a small proportion of vacancies on the A site would surely have the same effect. Doping with $\mathrm{Li}$ and $\mathrm{Na}$, at least, does not cause any change in $T_{\mathrm{N}} .{ }^{50}$ The strong influence of cation size on the structural evolution in comparison with the lack of its influence on the antiferromagnetic ordering is further emphasized by comparison with $\mathrm{NaMnF}_{3}, \mathrm{RbMnF}_{3}$, and $\mathrm{NH}_{4} \mathrm{MnF}_{3}$. From the compilation of $\operatorname{Cox},{ }^{87}$ these have $T_{\mathrm{N}}=60,82$, and $84 \mathrm{~K}$, respectively (Bartolomé et al. ${ }^{88}$ gave $T_{\mathrm{N}}=75 \mathrm{~K}$ for $\mathrm{NH}_{4} \mathrm{MnF}_{3}$ ), but at these temperatures $\mathrm{NaMnF}_{3}$ and $\mathrm{NH}_{4} \mathrm{MnF}_{3}$ are orthorhombic ${ }^{45-47}$ due to octahedral tilting, while $\mathrm{RbMnF}_{3}$ remains metrically cubic, ${ }^{89}$ although the magnetic ordering should in principle produce some distortion. In other words, essentially as considered by Gibaud et al., ${ }^{1}$ the magnetic and structural transitions are independent processes, which happen to occur in a similar temperature range in $\mathrm{KMnF}_{3}$ only by coincidence.

A feature of phase IV, if the assignment of space group Pnma is correct, is that the lattice geometry measured at low temperatures appears to be tetragonal since the $a$ and $c$ lattice parameters for the Pnma setting ( $b$ and $a$ in the Pbnm setting) are indistinguishable. ${ }^{1,15}$ The shear strain $e_{4}$ depends on the difference between the values of $a$ and $c$ and is related to the tilting order parameters according to

$$
e_{4}=\frac{\frac{a}{\sqrt{2}}-a_{\mathrm{o}}}{a_{\mathrm{o}}}-\frac{\frac{c}{\sqrt{2}}-a_{\mathrm{o}}}{a_{\mathrm{o}}}=\frac{-\lambda_{5} q_{4}^{2}}{2 q_{2}^{2}\left(\lambda_{6}+\lambda_{7}\right)+C_{44}^{\mathrm{o}}}
$$

where $a_{\mathrm{o}}$ is the reference parameter for a cubic structure at the same temperature, $\lambda_{5}, \lambda_{6}$, and $\lambda_{7}$ are different coupling coefficients, $q_{2}$ is the order parameter for $M$-point tilting, $q_{4}$ is the order parameter for $R$-point tilting (see below), and $C_{44}^{\mathrm{o}}$ is 
the elastic constant of the reference cubic phase. ${ }^{55}$ There are two obvious explanations for this discrepancy: either the value of $\lambda_{5}$ is small, i.e., coupling between the shear strain $e_{4}$ and the $R$-point tilting is weak or the space group assignment is incorrect. A tetragonal alternative with both $R$ - and $M$-point tilting is $P 4_{2} / n m c$ and, like Pnma, this could only develop from a $\mathrm{Cmcm}$ parent structure by a first-order transition. ${ }^{44}$ Resolution in the primary diffraction data may be an issue; Kapusta et al..$^{32}$ included a monoclinic distortion in their fit to an $\mathrm{X}$-ray powder diffraction pattern collected at $12 \mathrm{~K}$.

\section{B. Elastic behavior}

Variations in the elastic properties can be understood purely in terms of the sequence of tilting transitions without influence from the magnetic ordering. Each of the $M$ - and $R$-point order parameters have three components, $q_{1}-q_{3}$ and $q_{4}-q_{6}$, respectively, and these couple with strains $e_{i}, i=1-6 . e_{1}-e_{3}$ are more conveniently given in symmetry-adapted form as $e_{\mathrm{t}}$, which is a tetragonal shear strain, $e_{\mathrm{o}}$, which is an orthorhombic shear strain, and $e_{\mathrm{a}}$, which is the volume strain (see, for example, Refs. 70 and 90). Softening of the symmetry-adapted elastic constants as $T \rightarrow T_{\mathrm{c} 1}$ from above is consistent with the typical role of fluctuations ahead of a tilting transition driven by an $R$-point soft mode (I $\rightarrow$ II). The dominant effect is seen in $\frac{1}{2}\left(C_{11}-C_{12}\right)$, implying that the local fluctuations involve mainly coupling with a local tetragonal shear strain. Softening immediately below the transition is consistent with a conventional strain/order parameter coupling model for a transition that is close to being tricritical. ${ }^{35}$ The only nonzero order parameter, $q_{4}$, couples with the tetragonal shear strain, $e_{\mathrm{t}}$, as $\lambda e_{\mathrm{t}} q_{4}^{2}$, and the result is a sharp minimum in $\frac{1}{2}\left(C_{11}-C_{12}\right)$, seen separately also in $C_{11}$. The step in $C_{44}$ arises from a coupling term of the form $\lambda e_{4} q_{4} q_{6}$ [Fig. 1(a)], and the small and negligible step in $K$ [Fig. 1(a)] implies only very weak coupling (small value of $\lambda$ ) with the volume strain arising from $\lambda e_{\mathrm{a}} q_{4}^{2}$.

The $\mathrm{Cmcm}$ structure, proposed here for phase III, has nonzero-order-parameter components $q_{3}$ ( $M$ point) and $q_{4}(R$ point). ${ }^{44,90,91}$ Similar softening to that observed for the $R$-point transition (I $\rightarrow$ II) is expected ahead of the $M$-point transition (II $\rightarrow$ III), again with the main effect seen in $\frac{1}{2}\left(C_{11}-C_{12}\right)$ due to coupling of fluctuations with the local shear strains. Softening below the transition will have the same form for $\frac{1}{2}\left(C_{11}-C_{12}\right)$ as seen at the $R$-point transition because $q_{3}$ coupling with $e_{\mathrm{t}}$ and $e_{\mathrm{o}}$ also has the form, $\lambda e q_{3}^{2}$. The form of softening for $C_{44}$ will be different, however, because the $R$-point order parameter couples with $e_{4}$ as $\lambda e_{4} q_{4} q_{6}$ (plus related terms for $\left.e_{5}, e_{6}\right)$ and the $M$-point order parameter couples as $\lambda e_{4}^{2} q_{3}^{2}$ (plus related terms for $e_{5}, e_{6}$; e.g., see Refs. 55 and 90). As a result, $C_{44}$ must soften (or stiffen) in proportion to $q^{2}$, and there is only a step at $T_{\mathrm{c} 2}$ if the transition is first order. By way of sharp contrast, there should merely be discontinuities in all the elastic constants at the III $\left(0,0, q_{3}, q_{4}, 0,0\right) \rightarrow$ IV $\left(0, q_{2}, 0, q_{4}, 0, q_{6} ; q_{4}=q_{6}\right)$ transition because it is necessarily first order due to the change in orientation of tilt axes. Separate single-crystal data are not yet available for the full sequence in $\mathrm{KMnF}_{3}$, but the $C_{11}$ and $C_{44}$ data of Schranz et al ${ }^{35}$ for a sample with $1.8 \%$ Ca replacing $\mathrm{K}$ show the expected pattern quite clearly through all three transitions. These are reproduced in Fig. 9. In particular, $C_{44}$ softens continuously below $T_{\mathrm{c} 2}$, consistent with a transition which is thermodynamically continuous or, at least, almost so. Data from the present study are for mixtures of $\frac{1}{2}\left(C_{11}-C_{12}\right)$ and $C_{44}$, and the change in slope at $T_{\mathrm{c} 2}$ [Fig. $\left.6(\mathrm{~b})\right]$ must be due, at least in part, to the same softening of $C_{44}$ as seen in the doped sample. There certainly seems to be a sharp discontinuity at $T_{\mathrm{c} 3}$ (Fig. 6 for $\mathrm{KMnF}_{3}$, this study; $C_{11}$ of the doped sample of Schranz et al. ${ }^{35}$ reproduced in Fig. 9). There is only a minimal change in $C_{44}$ below $T_{\mathrm{c} 3}$ for the Ca-doped sample of Schranz et al., ${ }^{35}$ as would be expected for small (possibly zero) values of $e_{4}$. These strain and elasticity data are mutually consistent but do not discriminate between $P 4_{2} / n m c$ and $P$ nma (or $P 2_{1} / m$ ) as the correct space group for phase IV.

Nowhere in the elasticity data produced here or in the literature is there any evidence for anomalies in the shear elastic constants due to the magnetic ordering in $\mathrm{KMnF}_{3}$. If this is correct, the implication is that the magnetic order parameter does not couple significantly with any shear strain. A common strain mechanism is the most likely process by which the magnetic, $m$, and tilting order parameters, $q$, would couple so that the only common strain by which this could occur would be the volume strain. It would be unusual if there was no volume strain accompanying the magnetic ordering so that there must be at least some biquadratic coupling, $\lambda m^{2} q^{2}$, arising from the combination of $\lambda e_{\mathrm{a}} m^{2}$ and $\lambda e_{\mathrm{a}} q^{2}$. This will be weak if the volume strains are small. Proof of this weak or absent coupling behavior would require investigation of the strain and elastic properties of a crystal with $T_{\mathrm{c} 2}$ as far from $T_{\mathrm{N}}$ as possible, or through comparison with the elastic properties of closely related phases such as $\mathrm{NaMnF}_{3}$, $\mathrm{RbMnF}_{3}$, and $\mathrm{NH}_{4} \mathrm{MnF}_{3}$. For a sample with $T_{\mathrm{c} 2} \approx 94 \mathrm{~K}$, there indeed appears to be no sign of any significant strain arising at $T_{\mathrm{N}}{ }^{28}$ The only other phase for which elasticity data appear to be available is $\mathrm{RbMnF}_{3}$. This remains cubic through $T_{\mathrm{N}},{ }^{89}$ signifiying that the magnetic structure by itself does not induce a measurable symmetry-breaking shear strain. A small anomaly in longitudinal acoustic velocities ${ }^{92}$ indicates a weak coupling of the magnetic order parameter with the volume strain, however, which is seen also as a small anomaly in thermal expansion. ${ }^{89}$ A lack of coupling between the magnetic and structural order parameters is again consistent with the view of Gibaud et al. ${ }^{1}$ that it is purely a matter of coincidence that the values of $T_{\mathrm{N}}$ and $T_{\mathrm{c} 2}$ can be the same in $\mathrm{KMnF}_{3}$.

This lack of coupling between structural and magnetic order parameters may be a general feature of perovskites with octahedral tilting. For example, there is no evidence either of any shear strain or of any elastic anomaly accompanying the antiferromagnetic transition in crystals of $\mathrm{Pr}_{0.48} \mathrm{Ca}_{0.52} \mathrm{MnO}_{3} .{ }^{60,93}$ It is perhaps not surprising, therefore, that the variations in magnetic properties of $\mathrm{KMnF}_{3}$ attributed to movement of magnetic domain walls or changes in spin orientation below $\sim 60-70 \mathrm{~K}^{28,48,49}$ do not appear to have any influence on the elastic properties determined in the present study. The only possible correlation is that the suggested spin reorientation occurs at about the same temperature as $Q^{-1}$ becomes almost constant at relatively low values. If the two effects are related, a possible implication is that fluctuations of the octahedral tilting configuration or of twin-wall thicknesses and mobility close to $T_{\mathrm{c} 3}$ are sufficient to change the most 
TABLE II. Magnetic subgroups of Pnma1' to represent possible magnetic ordering schemes in $\mathrm{KMnF}_{3} . G, C$, and $A$ refer to different patterns of antiferromagnetic ordering for the components in the indicated crystallographic directions; F represents ferromagnetic ordering (following Cox in Ref. 87).

\begin{tabular}{lcccc}
\hline \hline & $\begin{array}{l}{[100]_{\text {Pnma }}} \\
{[010]_{\text {Pbnm }}} \\
{[011]_{\text {cubic }}}\end{array}$ & $\begin{array}{l}{[010]_{\text {Pnma }}} \\
{[001]_{\text {Pbnm }}} \\
{[100]_{\text {cubic }}}\end{array}$ & $\begin{array}{l}{[001]_{\text {Pnma }}} \\
{[100]_{\text {Pbnm }}} \\
{[01 \overline{1}]_{\text {cubic }}}\end{array}$ \\
Irrep & Space group & $G$ & $C$ & $A$ \\
$m \Gamma_{1}^{+}$ & $P n m a$ & $C$ & $G$ & $F$ \\
$m \Gamma_{2}^{+}$ & $P n^{\prime} m^{\prime} a$ & $F$ & $A$ & $C$ \\
$m \Gamma_{3}^{+}$ & $P n m^{\prime} a^{\prime}$ & $A$ & $F$ & $G$ \\
$m \Gamma_{4}^{+}$ & $P n^{\prime} m a^{\prime}$ & $A$ & \\
\hline \hline
\end{tabular}

stable spin configuration. (As an aside, the generality of limited coupling between magnetic ordering and octahedral tilting may be challenged as high-resolution diffraction data are collected for perovskites with Jahn-Teller active cations, including $\mathrm{Sr}_{0.65} \mathrm{Pr}_{0.35} \mathrm{MnO}_{3}$, for example. ${ }^{94}$ )

Both $f^{2}$ and $Q^{-1}$ level off in phase IV as $T \rightarrow 0 \mathrm{~K}$ [Fig. 6(a)], as expected for saturation of a single Einstein oscillator. ${ }^{95-99}$

\section{Magnetic behavior}

There are no symmetry constraints on the orientations of magnetic moments in the antiferromagnetically ordered structure of a perovskite with the crystallographic space group Pnma. Space groups for the possible magnetic structures can be identified in terms of the relevant irreducible representations (irreps), which describe their transformation properties. ISOTROPY has been used to identify both the irreps and space groups for the relevant magnetic subgroups of Pnma $1^{\prime}$ and $C m c m 1^{\prime}$, and these are given in Tables II and III. Following Cox ${ }^{87} G, C$, and $A$ are used to indicate the different ordering schemes of components of the magnetic moments along the crystallographic $x, y$, and $z$ directions as indicated, and $F$ shows a ferromagnetic component. Also given, for completeness, are the orientations of axes for the Pbnm setting of Pnma and the directions of pseudocubic axes. Heeger et al. ${ }^{16}$ reported that the observed ferromagnetic moment of the Pbnm structure is either along the crystallographic $x$ axis or along the $z$ axis, implying space groups $P n^{\prime} m^{\prime} a$ or $P n^{\prime} m a^{\prime}$. The former appears to be correct for the magnetic structure of $\mathrm{BaPrO}_{3} .{ }^{100}$

TABLE III. Magnetic subgroups of $C m \mathrm{~cm}^{1}{ }^{\prime}$ to represent possible magnetic ordering schemes in $\mathrm{KMnF}_{3} . G, C$, and $A$ refer to different patterns of antiferromagnetic ordering for the components in the indicated crystallographic directions; $F$ represents ferromagnetic ordering.

\begin{tabular}{ccccc}
\hline \hline Irrep & Space group & $\begin{array}{c}{[100]_{\mathrm{Cmcm}}} \\
{[100]_{\text {cubic }}}\end{array}$ & $\begin{array}{c}{[010]_{\mathrm{Cmcm}}} \\
{[00 \overline{1}]_{\text {cubic }}}\end{array}$ & $\begin{array}{c}{[001]_{\mathrm{Cmcm}}} \\
{[010]_{\text {cubic }}}\end{array}$ \\
\hline$m \Gamma_{1}^{+}$ & $C m c m$ & $A$ & $G$ & $C$ \\
$m \Gamma_{2}^{+}$ & $C m^{\prime} c^{\prime} m$ & $G$ & $A$ & $F$ \\
$m \Gamma_{3}^{+}$ & $C m c^{\prime} m^{\prime}$ & $F$ & $C$ & $G$ \\
$m \Gamma_{4}^{+}$ & $C m^{\prime} c m^{\prime}$ & $C$ & $F$ & $A$ \\
\hline \hline
\end{tabular}

The only purely antiferromagnetic subgroup of $\mathrm{Cmcm}^{\prime}$ is $\mathrm{Cmcm}$ (Table III). Given the apparent lack of coupling between order parameters for the magnetic and structural transitions, the factors influencing which magnetic ordering scheme is most stable are presumably due to quite subtle changes in the interactions between adjacent $\mathrm{Mn}^{2+}$ ions when the octahedral tilt system is changed. This is consistent with the only very small change in intensities of magnetic ordering reflections at the III $\leftrightarrow$ IV transition observed by Hidaka et al. ${ }^{25}$ Similarly, there does not appear to be any obvious symmetry argument as to why there might be a change in preferred orientation of the magnetic moments if there is a spin-reorientation transition below $\sim 50 \mathrm{~K}$ in $\mathrm{KMnF}_{3}$, as proposed by Saiki and Yoshioka, ${ }^{50}$ and below $\sim 70 \mathrm{~K}$ in Li- or Na-doped crystals. ${ }^{50}$ Thermodynamically continuous antiferromagnetic ordering occurs below $T_{\mathrm{N}}=87-88 \mathrm{~K}$ (e.g., see Fig. 1 of Ref. 17 and Fig. 4 of Ref. 25) independently of the octahedral tilting behavior, with changes at $82-84 \mathrm{~K}$ being due only to the fact that a canted ordering scheme is the lowest energy configuration in the Pnma structure, while the uncanted ordering scheme is preferred in the $\mathrm{Cmcm}$ structure. This conclusion follows the view already presented by Maartense and Searle ${ }^{22}$ that the observed magnetic behavior at $T_{\mathrm{c} 3}$ is the consequence of rather than the cause of the structural evolution.

\section{Domain-wall dynamics}

Given that the elastic anomalies can be attributed essentially to the structural evolution alone, it is likely that the anelastic loss behavior is due exclusively to the mobility of crystallographic defects. Differences as large as $\sim 17 \%$ between the relaxed and unrelaxed elastic constants are also consistent with the defects responsible being ferroelastic twin walls in the tetragonal phase. The easy mobility of twin walls gives rise to significantly greater shear strains than can occur by simply bending the crystal. A common pattern is of a peak in attenuation just below the transition temperature, a steady decrease with further decrease in temperature followed by a large peak and then a return to low levels of dissipation. This is seen, for example, in $\mathrm{LaAlO}_{3}{ }^{101}$ Immediately below the transition temperature there are variations in twin-wall thicknesses such that, with decreasing temperature, the wall thickness decreases. ${ }^{102}$ The wall mobility is greatest for thick walls and decreases with decreasing wall thickness (e.g., see Ref. 103). At some low temperature the walls then become pinned by defects. The present data show essentially this pattern, except that values of $Q^{-1}$ do not return to their lowest values below the peak at $\sim 130 \mathrm{~K}$ (Figs. 5 and 6 ).

As with twin-wall-related anelasticity in $\mathrm{LaAlO}_{3},{ }^{58}$ the observed behavior of $\mathrm{KMnF}_{3}$ at frequencies of a few hertz does not match up with the behavior at 0.1-1 MHz. Extrapolating the data in Fig. 2 of Salje and Zhang ${ }^{37}$ from $0.2-32 \mathrm{~Hz}$ to $\sim 0.1 \mathrm{MHz}$ would give freezing temperatures in the vicinity of $250 \mathrm{~K}$ rather than $\sim 130 \mathrm{~K}$. This requires that a different loss mechanism applies under the high frequency and relatively low stress conditions of RUS in comparison with the low-frequency and relatively high-stress conditions of a DMA experiment. Carpenter $e t$ al. ${ }^{58}$ have speculated that the difference is between movement of the tips of needle domains (low frequencies) and of local bowing of segments of the 
twin walls (high frequencies). A local mechanism could be by displacement of ledges within the twin walls. ${ }^{104}$ These might also be referred to as kinks or latches inside the wall that move laterally. With each transfer from one surface to the opposite surface, there would be an advance of the twin wall by the height of the kink, as has been investigated in simulations. ${ }^{105}$ Such kinks would be destabilized for large wall thicknesses near the transition point.

Equation (6) should be applied, strictly, to measurements of $Q^{-1}$ made at constant frequency rather than across a spread of frequencies, but the fits at least imply activation energy barriers to twin-wall motion in the vicinity of $\sim 16 \mathrm{~kJ}$. mole $^{-1}$ or higher, depending on the spread of pinning energies. These are in the same range as reported previously for twin-wall pinning in $\mathrm{KMnF}_{3}$ and $\mathrm{K}_{0.997} \mathrm{Ca}_{0.003} \mathrm{MnF}_{3}\left(\sim 13-41 \mathrm{~kJ} . \mathrm{mole}^{-1}{ }^{35,37}\right)$. By comparison with the energies calculated for defects, ${ }^{106,107}$ the pinning process perhaps involves $F$ vacancies ${ }^{37}$ or dumbbell pairs of $F$ interstitials. ${ }^{35}$ The fact that $Q^{-1}$ does not return to values as low as those for the cubic phase or for phase IV at low temperatures implies, perhaps, that not all the twin walls become pinned. Salje and Zhang ${ }^{37}$ have speculated that the apparently low level of pinning encountered in low-frequency measurements is due to a low concentration of the relevant defects or to rather weak pinning. This is reinforced by the attenuation data of Fossheim et al. ${ }^{24}$ at 11.7 $\mathrm{MHz}$ for an orientation (longitudinal acoustic waves parallel to [100]), which would have delivered some shear stress on the twin walls. Using $\tau_{\mathrm{o}}=8 \times 10^{-13} \mathrm{~s}$ and $E_{\mathrm{a}}=$ $16 \mathrm{~kJ} . \mathrm{mole}^{-1}$ in Eq. (3) gives an expected freezing temperature of $\sim 200 \mathrm{~K}$ at $12 \mathrm{MHz}$, implying that their experiment was probably conducted below the freezing point of the twin walls. Significant attenuation was, nevertheless, observed and could certainly have arisen from local displacements of twin walls, which were only weakly pinned or were not pinned at all.

In their measurements on $\mathrm{KMnF}_{3}$ at $10 \mathrm{MHz}$, Maartense and Searle $^{22}$ observed marked acoustic attenuation below $T_{\mathrm{c} 1}$, which decreased abruptly below $T_{\mathrm{c} 3}$. This matches the results reported here for $\sim 0.1-1 \mathrm{MHz}$ (Fig. 5). High mobility of purely ferroelastic twin walls appears to be typical of tetragonal and rhombohedral perovskites, including ( $\mathrm{Ca}, \mathrm{Sr}) \mathrm{TiO}_{3},{ }^{72,75,104,108-111}$ ( $\left.\mathrm{La}, \mathrm{Pr}\right) \mathrm{AlO}_{3},{ }^{59,63,112,113}$ $(\mathrm{K}, \mathrm{Ca}) \mathrm{MnF}_{3},{ }^{35,37,64,65,114} \quad(\mathrm{Sr}, \mathrm{Ba}) \mathrm{SnO}_{3},{ }^{73,74} \quad$ and $\mathrm{Sr}(\mathrm{Ti}, \mathrm{Zr}) \mathrm{O}_{3} .{ }^{55,56,61}$ By way of contrast, twin walls in Pnma perovskites generally do not appear to be mobile under the application of an external stress. ${ }^{55,56,72,73,75}$ One possible explanation suggested for the different characteristics of the latter relates to the role of volume strain in influencing the thickness of the twin walls and, hence, their interaction with defects. ${ }^{73}$ Another is that the walls might actually be mobile, but the strain contrast across them is too small to give obvious softening and anelastic loss. $^{70,72}$ A more general explanation might relate to the fact that the twin walls in Pnma perovskites depend on two order parameters with quite different symmetries and that this somehow results in them being jammed. ${ }^{55}$ This last explanation can now be ruled out as a generality since phase III of $\mathrm{KMnF}_{3}$ has both $M$-point and $R$-point tilting but still shows anelastic loss; the abrupt difference is between phase III $(\mathrm{Cmcm})$ and phase IV (Pnma). Moreover, it has recently been found that the $P$ nma phase of $\mathrm{BaCeO}_{3}$ displays strong anelastic losses at both high and low frequencies. ${ }^{62}$ One systematic cor- relation is that the $R$-point tilted structure of $\mathrm{BaCeO}_{3}$ is rhombohedral $(R \overline{3} c)$, while the others are all tetragonal $(I 4 / \mathrm{mcm})$, but this might be fortuitous. Factors that determine these particular characteristics of twin-wall dynamics remain to be identified, therefore. There is still some small increase in $Q^{-1}$ as the $\mathrm{III} \leftrightarrow \mathrm{IV}$ transition is approached from below [Fig. 6(a)], which is similar to that observed at the Imma $\leftrightarrow$ Pnma transition in $\mathrm{SrZrO}_{3}{ }^{55}$ and is probably indicative of precursor fluctuations.

Additional variations of $Q^{-1}$ recorded by selected acoustic resonances in the stability field of the cubic structure (Fig. 5) have not been fully characterized here but are presumably due to other types of defects present in the $\mathrm{KMnF}_{3}$ structure or are related to the presence of the birefringent particles identified tentatively as $\mathrm{MnF}_{2}$.

\section{E. Magnetoelastic coupling and manipulation of twin orientations}

It has been concluded here that the magnetic order parameter does not couple with shear strain, and it follows that there will not be a strain contrast across purely magnetic domain walls that might result in their motion under the application of a shear stress. On the other hand, there is a preferred crystallographic orientation of the weak ferromagnetic moment in phase IV so that application of a strong magnetic field could lead to reorientation of the ferroelastic twins. This allows, in principle, for some manipulation of magnetic properties. Although these were not explored systematically, the hysteresis measurements reported in Fig. 3 show that fields up to $15 \mathrm{kOe}$ can cause a substantial change in the magnetic susceptibility of phase IV. The hysteresis cycles indicate normal changes in configuration of the magnetic domains, but the before and after differences indicate that there has probably been some change also in crystallographic topology through reorientation of the ferroelastic twins.

Changes in susceptibility between cooling and heating in (nominally) zero field (Fig. 2) can also be understood simply in terms of changes in ferroelastic twin configurations. During cooling through $T_{\mathrm{c} 1}$ an equal proportion of the three possible ferroelastic twin orientations would appear. If there is any anisotropy in paramagnetic susceptibility of the $I 4 / \mathrm{mcm}$ structure, it will not be observed because of averaging over all the twin configurations. On cooling through the II $\rightarrow$ III and III $\rightarrow$ IV transition, new sets of twins will develop, and these will not necessarily be present in random orientations, particularly if the magnetic field has any influence. On reversing the sequence, i.e., IV $\rightarrow$ III $\rightarrow$ II, the twins present in the $I 4 / \mathrm{mcm}$ structure will depend on the previous magnetoelastic history, and all orientations need not be present in equal proportions. Any anisotropy of the paramagnetic susceptibility would then give rise to differences between the values measured during heating and cooling-just as is observed [Fig. 2(c)]. In a limiting case it might be possible to produce an elastically poled tetragonal crystal, i.e., with one ferroelastic twin orientation, by cooling into the stability field of phase IV, applying a strong field, and then heating back to the stability field of phase II with the field kept in place. The corollary of this is that such a poled crystal would exhibit a change in net ferromagnetic moment if subjected to a shear stress that induced some reorientation of ferroelastic twins. 
An additional feature of the magnetic hysteresis highlighted in Fig. 2(c) is that the break in slope during heating, which would signify a change in paramagnetic anisotropy, occurs at $\sim 200 \mathrm{~K}$ rather than at $186 \mathrm{~K}$. This is much greater than the hysteresis of $0.2 \mathrm{~K}$ for $T_{\mathrm{c} 1}$ due to the first-order character of the transition. ${ }^{11}$ The difference in structural states between cooling and heating is also sufficient at $189 \mathrm{~K}$ to give marked differences in elastic properties, as shown by the frequencies of individual peaks in the RUS spectra, but the difference has almost disappeared at $220 \mathrm{~K}$ (Fig. 10). Some parts of the crystal must retain local noncubic geometry well into the stability field of the cubic structure, and a tweed microstructure with some preferred orientation is the most likely cause of this. Strain fields around the inclusions might also play a part in producing local anisotropy, however. A tweed microstructure could also be a contributory factor to either or both of the narrow and broad components of a central peak observed in inelastic neutron scattering spectra between $T_{\mathrm{c} 1}$ and $\sim 210 \mathrm{~K}$ by Shapiro et al. ${ }^{115}$ Evidence of short-range ordering up to 215.5 K has also been observed in infrared spectra, with the suggestion that defects would help to stabilize a tweed microstructure. ${ }^{36}$

The combination of ferroelastic properties arising from octahedral tilting plus magnetism in $\mathrm{KMnF}_{3}$ makes an interesting contrast with $\mathrm{PrAlO}_{3}$, where there is one tilt system but additional ferroelastic twinning due to a Jahn-Teller distortion (Refs. 116,117, and references therein). Twin-wall-related anelasticity in the stability field of the $R \overline{3} c$ structure of $\mathrm{PrAlO}_{3}$ is essentially the same as seen in $I 4 / \mathrm{mcm}$ perovskites, but twin

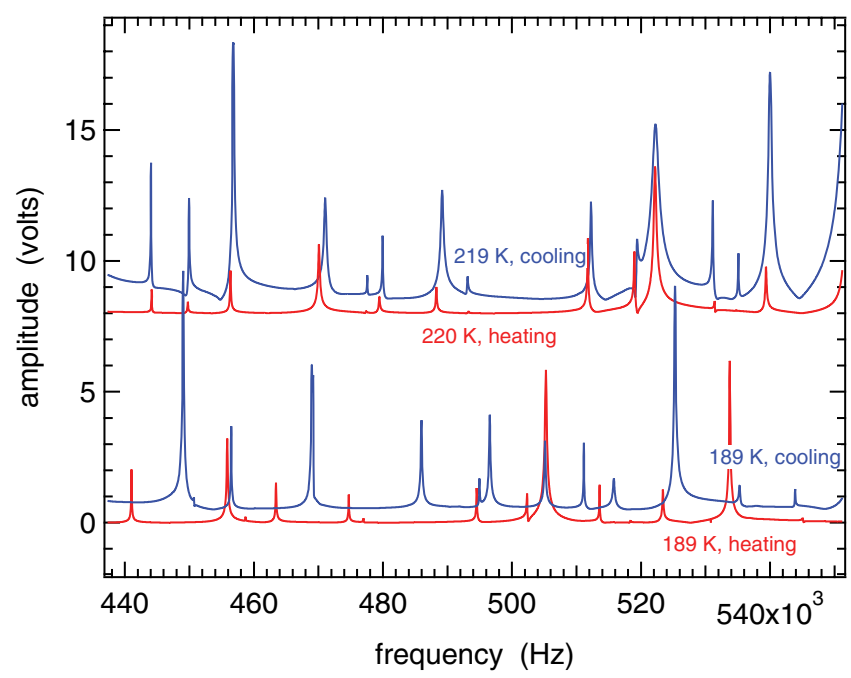

FIG. 10. (Color online) RUS spectra collected at 189 and $220 \mathrm{~K}$ during heating and cooling show a distinct hysteresis in peak positions. Peaks in the spectra collected at $189 \mathrm{~K}$ have markedly different frequencies, but the difference between heating and cooling is small at $220 \mathrm{~K}$. This corresponds to a significant difference of the bulk elastic properties between the crystal prior to being cooled through $T_{\mathrm{cl}}$ and after being heated from $\sim 10 \mathrm{~K}$. It is interpreted as signifying the presence of a tweed microstructure in a small temperature interval above $T_{\mathrm{c} 1}$. On cooling, the tweed will develop with all possible orientations, but during heating some orientations of tweed may develop preferentially from a tetragonal crystal with preferred orientations of twin domains. walls of the monoclinic phase appear also to be highly mobile below the Imma $\leftrightarrow C 2 / m$ transition at $151 \mathrm{~K} .{ }^{59,63}$ Crystals remain paramagnetic, but the easy mobility of twin walls then appears to allow easy reorientation of ferroelastic domains by application of a magnetic field. ${ }^{59,118}$ In other words, the dynamics of switching in a magnetic field are determined by the dynamics of ferroelastic twin-wall motion, and this can be selected on the basis of the underlying mechanism for the ferroelastic phase transition.

\section{F. Magnetic properties of twin walls}

One of the potentially fruitful avenues of investigation in multiferroic materials relates to the possibility of engineering materials with domain walls which have quite different properties from the matrix in which they reside. ${ }^{119,120}$ In the simplest analysis the structure of a transformation twin wall or antiphase boundary is that of the parent structure above the transition point at which the domains developed. As shown for the case of ferrielectric twin walls in the paraelectric matrix of $\mathrm{CaTiO}_{3}$, however, this is not always the case. ${ }^{121,122}$ The existence of multiple order parameters allows the possibility of additional relaxations that do not belong to either of the parent or product phases. Interest here would be in magnetic properties of the ferroelastic twin walls.

From the simplest perspective, the structure of twin walls in a crystal with one tilt system (phase II) would be expected to be essentially that of the cubic parent. Magnetic moments of the expected ( $G$-type) antiferromagnetic ordering in the matrix are constrained by symmetry to lie parallel to one of the crystallographic axes (Table IV). If magnetic ordering occurred in the twin walls and cubic lattice geometry was maintained, a supercell with the dimensions $2 a \times 2 a \times 2 a$ would be required, and a complicated distribution of spin orientations would be required to maintain cubic symmetry. A selection of magnetic subgroups of $P m \overline{3} m 1^{\prime}$ is listed in Table V, with the simplest $G, C, A$ (antiferromagnetic), and $F$ (ferromagnetic) ordering schemes, to emphasize that cubic lattice geometry could only be maintained if coupling of the magnetic order parameter with a tetragonal shear strain was negligibly small. Magnetic ordering in $\mathrm{RbMnF}_{3}$ is given by Pickart et al. ${ }^{123}$ as $G$-type, and the structure is found to be metrically cubic, ${ }^{89}$ so perhaps this provides the best guide to how the twin walls in phase II of $\mathrm{KMnF}_{3}$ would order. Below $T_{\mathrm{N}}$, this would give antiferromagnetic twin walls in an antiferromagnetic matrix.

TABLE IV. Magnetic subgroups of $I 4 / \mathrm{mcm}^{\prime}$ to represent possible magnetic ordering schemes in $\mathrm{KMnF}_{3}$. $G$ refers to a pattern of antiferromagnetic ordering in a given crystallographic direction; $F$ represents ferromagnetic ordering.

\begin{tabular}{lcccc}
\hline \hline Irrep & SG & $\begin{array}{l}{[100]_{\mathrm{I} / \mathrm{mcm}}} \\
{[110]_{\text {cubic }}}\end{array}$ & $\begin{array}{l}{[010]_{\mathrm{I} 4 / \mathrm{mcm}}} \\
{[\overline{1} 10]_{\text {cubic }}}\end{array}$ & $\begin{array}{c}{[001]_{\mathrm{I} / \mathrm{mcm}}} \\
{[001]_{\mathrm{cubic}}}\end{array}$ \\
\hline$m \Gamma_{1}^{+}$ & $I 4 / m c m$ & - & - & $G$ \\
$m \Gamma_{3}^{+}$ & $I 4 / m c^{\prime} m^{\prime}$ & - & - & $F$ \\
$m \Gamma_{5}^{+}(\mathrm{a}, 0)$ & $I b a^{\prime} m^{\prime}$ & $F$ & $G$ & - \\
$m \Gamma_{5}^{+}(\mathrm{a}, \mathrm{a})$ & $F m^{\prime} m^{\prime} m$ & & & \\
$m \Gamma_{5}^{+}(\mathrm{a}, \mathrm{b})$ & $C 2^{\prime} / m^{\prime}$ & & & \\
\hline \hline
\end{tabular}


TABLE V. Magnetic subgroups of $P m \overline{3} m 1^{\prime}$ for four simple ordering schemes in $\mathrm{KMnF}_{3}$. Subgroups of the true cubic symmetry required more complicated patterns of ordering in a $2 a \times 2 a \times 2 a$ unit supercell.

\begin{tabular}{ccccc} 
Irrep & Order parameter components & $\mathrm{SG}$ & Ordering scheme & Basis vectors [origin $(0,0,0)]$ \\
\hline$m \Gamma_{4}^{+}$ & $(\mathrm{a}, 0,0)$ & $P 4 / m m^{\prime} m^{\prime}$ & $F$ & $(0,1,0),(0,0,1),(1,0,0)$ \\
$m \mathrm{X}_{3}^{+}$ & $(\mathrm{a}, 0,0)$ & $P_{c} 4 / m c c$ & $A$ & $(1,0,0),(0,0,1),(0,-2,0)$ \\
$m \mathrm{M}_{3}^{+}$ & $(\mathrm{a}, 0,0)$ & $P_{C} 4 / m b m$ & $C$ & $(1,-1,0),(1,1,0),(0,0,1)$ \\
$m \mathrm{R}_{4}^{+}$ & $(\mathrm{a}, 0,0)$ & $I_{c} 4 / m c m$ & $G$ & $(-1,1,0),(-1,-1,0),(0,0,2)$ \\
\hline \hline
\end{tabular}

Phase III of $\mathrm{KMnF}_{3}$ would have twin walls inherited from the $P m \overline{3} m \leftrightarrow I 4 / m c m$ transition and new twin walls arising from the $I 4 / m c m \leftrightarrow C m c m$ transition. The latter would be expected to have the structure of the $I 4 / \mathrm{mcm}$ structure, but additional relaxations might also occur. Below $T_{\mathrm{N}}$ the matrix would be antiferromagnetic, but ferromagnetic twin walls are conceivable, depending on the locally preferred magnetic ordering scheme if the symmetry of the twin walls deviates from $I 4 / \mathrm{mcm}$. The greatest possibilities for differences in magnetic structure between twins and twin walls are certainly in the Pnma structure, however. The starting point for a search to produce ferromagnetic walls in an antiferromagnetic matrix would be for a Pnma perovskite, with $\mathrm{Fe}, \mathrm{Co}, \mathrm{Ni}, \mathrm{Mn}$, or $\mathrm{Cr}$, for example, in which the preferred magnetic ordering scheme of the matrix has the symmetry properties of irrep $m \Gamma_{1}^{+}$. Twin walls with locally preferred ordering schemes corresponding to $m \Gamma_{2}^{+}, m \Gamma_{3}^{+}$, or $m \Gamma_{4}^{+}$would be ferromagnetic due to canting.

\section{CONCLUSIONS}

Octahedral tilting and magnetic ordering transitions in $\mathrm{KMnF}_{3}$ arise by entirely separate mechanisms with little or no coupling. In particular, the observed strain, elastic, and anelastic behavior is understandable entirely in terms of a sequence of structural states with two different instabilities relating to $R$-point and $M$-point tilting. There is a single magnetic instability, but the preferred magnetic ordering schemes do not have a measurable coupling with shear strain. Interesting combinations of magnetic and ferroelastic properties are due effectively to the coincidence that the critical temperature for $M$-point tilting, near $T_{\mathrm{c} 2}$, is close to the critical temperature for antiferromagnetic ordering, $T_{\mathrm{N}}$.

There are potentially two genuinely multiferroic transitions. The first is at $T_{\mathrm{c} 2}$ for crystals with compositions and defect contents that have $T_{\mathrm{c} 2}=T_{\mathrm{N}}$. This involves the simultaneous development of ferroelastic and antiferromagnetic properties. Coupling between the magnetic and structural order parameters is likely to be primarily biquadratic, depending on coupling with a common volume strain. The second genuinely multiferroic transition, with simultaneous changes in both magnetic and ferroelastic properties, occurs at $T_{\mathrm{c} 3}$. This is not due to a confluence of separate instabilities, however, but follows simply from the fact that the preferred magnetic ordering scheme is different between Pnma (weakly ferromagnetic) and Cmcm (antiferromagnetic) structures. The driving mechanism for the transition is a change in octahedral tilt axes. Changes in composition can be used to manipulate the structural transition temperatures according to conventional Goldschmidt tolerance factor arguments, while leaving $T_{\mathrm{N}}$ essentially unchanged. Understanding of these relationships opens up the possibility of engineering crystals with manipulable combinations of magnetic and ferroelastic properties.

\section{ACKNOWLEDGMENTS}

The RUS facilities in Cambridge were established through a grant from the Natural Environment Research Council of Great Britain (Grant No. NE/B505738/1). Wilfried Schranz is thanked for providing the elastic constant data, which appear in Fig. 9.
*Corresponding author: mc43@esc.cam.ac.uk

${ }^{1}$ A. Gibaud, S. M. Shapiro, J. Nouet, and H. You, Phys. Rev. B 44, 2437 (1991).

${ }^{2}$ G. Shirane, V. J. Minkiewicz, and A. Linz, Solid State Commun. 8, 1941 (1970).

${ }^{3}$ K. S. Aleksandrov and L. M. Reshchikova, Sov. Phys. Crystallogr. 14, 614 (1970).

${ }^{4}$ S. Stokka, K. Fossheim, and V. Samulionis, Phys. Rev. Lett. 47, 1740 (1981).

${ }^{5}$ H. Sakashita, N. Ohama, and A. Okazaki, J. Phys. Soc. Jpn. 50, 4013 (1981).

${ }^{6}$ H. Sakashita, N. Ohama, and A. Okazaki, Phase Transit. 28, 99 (1990).

${ }^{7}$ S. Hirotsu and S. Sawada, Solid State Commun. 12, 1003 (1973).
${ }^{8}$ F. J. Romero, M. C. Gallardo, J. Jiménez, J. del Cerro, E. K. H. Salje, and A. Gibaud, Phase Transit. 68, 523 (1999).

${ }^{9}$ F. J. Romero, M. C. Gallardo, S. A. Hayward, J. Jiménez, J. del Cerro, and E. K. H. Salje, J. Phys.: Condens. Matter 16, 2879 (2004).

${ }^{10}$ M. C. Gallardo, F. J. Romero, S. A. Hayward, E. K. H. Salje, and J. del Cerro, Miner. Mag. 64, 971 (2000).

${ }^{11}$ S. A. Hayward, F. J. Romero, M. C. Gallardo, J. del Cerro, A. Gibaud, and E. K. H. Salje, J. Phys.: Condens. Matter 12 , 1133 (2000).

${ }^{12}$ S. Ogawa, J. Phys. Soc. Jpn. 14, 1115 (1959).

${ }^{13}$ R. G. Shulman, K. Knox, and B. J. Wyluda, Bull. Am. Phys. Soc. 4, 166 (1959).

${ }^{14}$ V. Scatturin, L. Corliss, N. Elliott, and J. Hastings, Acta Crystallogr. 14, 19 (1961). 
${ }^{15}$ O. Beckman and K. Knox, Phys. Rev. 121, 376 (1961).

${ }^{16}$ A. J. Heeger, O. Beckman, and A. M. Portis, Phys. Rev. 123, 1652 (1961).

${ }^{17}$ M. J. Cooper and R. Nathans, J. Appl. Phys. 37, 1041 (1966).

${ }^{18}$ M. Furukawa, Y. Fujimori, and K. Hirakawa, J. Phys. Soc. Jpn. 29, 1528 (1970).

${ }^{19}$ V. J. Minkiewicz, Y. Fujii, and Y. Yamada, J. Phys. Soc. Jpn. 28, 443 (1970).

${ }^{20}$ R. L. Melcher and R. H. Plovnik, in Phonons, edited by M. A. Nusimovici (Flammarion, Paris, 1971), p. 348.

${ }^{21}$ K. Saiki, J. Phys. Soc. Jpn. 33, 1284 (1972).

${ }^{22}$ I. Maartense and C. W. Searle, Phys. Rev. B 6, 894 (1972).

${ }^{23}$ F. Borsa, Phys. Rev. B 7, 913 (1973).

${ }^{24}$ K. Fossheim, D. Martinsen, and A. Linz, in Anharmonic Lattices, Structural Transitions and Melting, edited by T. Riste, Vol. 1 (Noordhof, Leiden, 1974), p. 141.

${ }^{25}$ M. Hidaka, N. Ohama, A. Okazaki, H. Sakashita, and S. Yamakawa, Solid State Commun. 16, 1121 (1975).

${ }^{26}$ D. J. Benard and W. C. Walker, Rev. Sci. Instrum. 47, 122 (1976).

${ }^{27}$ R. M. Holt and K. Fossheim, Phys. Rev. B 24, 2680 (1981).

${ }^{28}$ J. Bartolomé, J. A. Rojo, R. Navarro, D. Gonzalez, M. R. Ibarra, and A. del Moral, J. Magn. Magn. Mater. 31-34, 1052 (1983).

${ }^{29}$ M. Hidaka, H. Fujii, and S. Maeda, Phase Transit. 6, 101 (1986).

${ }^{30}$ M. Hidaka, Z. Y. Zhou, and S. Yamashita, Phase Transit. 18, 103 (1989).

${ }^{31}$ A. Ratuszna and A. Kachel, Acta Crystallogr. B 48, 118 (1992).

${ }^{32}$ J. Kapusta, P. Daniel, and A. Ratuszna, Phys. Rev. B 59, 14235 (1999).

${ }^{33}$ S. Watanabe, M. Yoshimura, H. Hidaka, and H. Yoshizawa, Appl. Phys. A 84, 87 (2006).

${ }^{34}$ A. Salazar, M. Massot, A. Oleaga, A. Pawlak, and W. Schranz, Phys. Rev. B 75, 224428 (2007).

${ }^{35}$ W. Schranz, P. Sondergeld, A. V. Kityk, and E. K. H. Salje, Phys. Rev. B 80, 094110 (2009).

${ }^{36}$ E. K. H. Salje, M. Zhang, and H. Zhang, J. Phys.: Condens. Matter 21, 335402 (2009).

${ }^{37}$ E. K. H. Salje and H. Zhang, J. Phys.: Condens. Matter 21, 035901 (2009).

${ }^{38}$ S. A. Kizhaev and L. A. Markova, Phys. Solid State 53, 1851 (2011).

${ }^{39}$ V. J. Minkiewicz and G. Shirane, J. Phys. Soc. Jpn. 26, 674 (1969).

${ }^{40}$ K. Gesi, J. D. Axe, G. Shirane, and A. Linz, Phys. Rev. B 5, 1933 (1972).

${ }^{41}$ A. Ratuszna, J. Phys.: Condens. Matter 5, 841 (1993).

${ }^{42}$ D. J. Lockwood and B. H. Torrie, J. Phys. C 7, 2729 (1974).

${ }^{43}$ M. Hidaka, J. Phys. Soc. Jpn. 39, 103 (1975).

${ }^{44}$ C. J. Howard and H. T. Stokes, Acta Crystallogr. B 54, 782 (1998).

${ }^{45}$ A. Katrusiak and A. Ratuszna, Solid State Commun. 84, 435 (1992).

${ }^{46}$ J. D. Tornero and J. Fayos, Ferroelectrics Lett. 12, 85 (1990).

${ }^{47}$ M. A. Laguna, M. L. Sanjuán, V. M. Orera, J. Rubín, E. Palacios, M. C. Piqué, J. Bartolomé, and J. F. Berar, J. Phys.: Condens. Matter 5, 283 (1993).

${ }^{48}$ I. Maartense, Solid State Commun. 12, 1133 (1973).

${ }^{49}$ K. Saiki and H. Yoshioka, Solid State Commun. 15, 1067 (1974).

${ }^{50}$ D. Skrzypek, J. Phys.: Condens. Matter 6, 6815 (1994).

${ }^{51}$ W. Eerenstein, N. D. Mathur, and J. F. Scott, Nature 442, 759 (2006).
${ }^{52}$ G. Catalan and J. F. Scott, Adv. Mater. 21, 2463 (2009).

${ }^{53}$ J. F. Scott and R. Blinc, J. Phys.: Condens. Matter 23, 113202 (2011).

${ }^{54}$ A. Migliori and J. L. Sarrao, Resonant Ultrasound Spectroscopy: Applications to Physics, Materials Measurements and Nondestructive Evaluation (Wiley, New York, 1997).

${ }^{55}$ R. E. A. McKnight, C. J. Howard, and M. A. Carpenter, J. Phys.: Condens. Matter 21, 015901 (2009).

${ }^{56}$ R. E. A. McKnight, B. J. Kennedy, Q. Zhou, and M. A. Carpenter, J. Phys.: Condens. Matter 21, 015902 (2009).

${ }^{57}$ J. Manchado, F. J. Romero, M. C. Gallardo, J. del Cerro, T. W. Darling, P. A. Taylor, A. Buckley, and M. A. Carpenter, J. Phys.: Condens. Matter 21, 295903 (2009).

${ }^{58}$ M. A. Carpenter, A. Buckley, P. A. Taylor, and T. W. Darling, J. Phys.: Condens. Matter 22, 035405 (2010).

${ }^{59}$ M. A. Carpenter, E. C. Wiltshire, C. J. Howard, R. I. Thomson, S. Turczynski, D. A. Pawlak, and T. Lukasiwicz, Phase Transit. 83, 703 (2010).

${ }^{60}$ M. A. Carpenter, C. J. Howard, R. E. A. McKnight, A. Migliori, J. B. Betts, and V. R. Fanelli, Phys. Rev. B 82, 134123 (2010).

${ }^{61}$ Z. Zhang, J. Koppensteiner, W. Schranz, and M. A. Carpenter, J. Phys.: Condens. Matter 22, 295401 (2010).

${ }^{62}$ Z. Zhang, J. Koppensteiner, W. Schranz, J. B. Betts, A. Migliori, and M. A. Carpenter, Phys. Rev. B 82, 014113 (2010).

${ }^{63}$ R. I. Thomson, J. M. Rawson, C. J. Howard, S. Turczynski, D. A. Pawlak, T. Lukasiewicz, and M. A. Carpenter, Phys. Rev. B 82, 214111 (2010).

${ }^{64}$ W. Schranz, A. Tröster, A. V. Kityk, P. Sondergeld, and E. K. H. Salje, Europhys. Lett. 62, 512 (2003).

${ }^{65}$ W. Schranz and A. V. Kityk, Ferroelectrics 375, 178 (2008).

${ }^{66}$ H. T. Stokes, D. M. Hatch, and B. J. Campbell, ISOTROPY (accessed March 2012), http://stokes.byu.edu/isotropy.html.

${ }^{67}$ L. M. Reshchikova, V. I. Zinenko, and K. S. Aleksandrov, Sov. Phys. Solid State 11, 2893 (1970).

${ }^{68}$ W. Cao and G. R. Barsch, Phys. Rev. B 38, 7947 (1988).

${ }^{69}$ J. C. Slonczewski and H. Thomas, Phys. Rev. B 1, 3599 (1970).

${ }^{70}$ M. A. Carpenter, Am. Mineral. 92, 309 (2007).

${ }^{71}$ M. A. Carpenter and E. K. H. Salje, Eur. J. Mineral. 10, 693 (1998).

${ }^{72}$ R. J. Harrison, S. A. T. Redfern, and J. Street, Am. Mineral. 88, 574 (2003).

${ }^{73}$ M. Daraktchiev, R. J. Harrison, E. H. Mountstevens, and S. A. T. Redfern, Mater. Sci. Eng. A 442, 199 (2006).

${ }^{74}$ M. Daraktchiev, E. K. H. Salje, W. T. Lee, and S. A. T. Redfern, Phys. Rev. B 75, 134102 (2007).

${ }^{75}$ J. N. Walsh, P. A. Taylor, A. Buckley, T. W. Darling, J. Schreuer, and M. A. Carpenter, Phys. Earth Planet. Inter. 167, 110 (2008).

${ }^{76}$ K. Fossheim and R. M. Holt, Phys. Rev. Lett. 45, 730 (1980).

${ }^{77}$ R. L. Melcher, Phys. Rev. B 2, 733 (1970).

${ }^{78}$ M. Guennou, P. Bouvier, G. Garbarino, J. Kreisel, and E. K. H. Salje, J. Phys.: Condens. Matter 23, 485901 (2011).

${ }^{79}$ R. E. A. McKnight, M. A. Carpenter, T. W. Darling, A. Buckley, and P. A. Taylor, Am. Mineral. 92, 1665 (2007).

${ }^{80}$ M. McElfrish, S. Li, and R. Sager, (accessed March 2012), www.qdusa.com/sitedocs/appNotes/mpms/effects.pdf.

${ }^{81}$ R. Schleck, Y. Nahas, R. P. S. M. Lobo, J. Varignon, M. B. Lepetit, C. S. Nelson, and R. L. Moreira, Phys. Rev. B 82, 054412 (2010).

${ }^{82}$ J. F. Nye, Physical Properties of Crystals (Oxford University Press, Oxford, 1985). 
${ }^{83}$ A. S. Nowick and B. S. Berry, Anelastic Relaxation in Crystalline Solids (Academic Press, New York, 1972).

${ }^{84}$ M. Weller, G. Y. Li, J. X. Zhang, T. S. Ke, and J. Diehl, Acta Metall. 29, 1047 (1981)

${ }^{85}$ R. Schaller, G. Fantozzi, and G. Gremaud, Mechanical Spectroscopy $Q^{-1}$ 2001: With Applications to Materials Science (Trans Tech. Publ., Clausthal, Germany, 2001).

${ }^{86}$ A. Ratuszna and J. Kapusta, Phase Transit. 62, 181 (1997).

${ }^{87}$ D. E. Cox, IEEE Trans. Magn. 8, 161 (1972).

${ }^{88}$ J. Bartolomé, R. Burriel, F. Palacio, D. Gonzalez, R. Navarro, J. A. Rojo, and L. J. de Jongh, Physica B 115, 190 (1983).

${ }^{89}$ D. T. Teaney, V. L. Moruzzi, and B. E. Argyle, J. Appl. Phys. 37, 1122 (1966).

${ }^{90}$ M. A. Carpenter, A. I. Becerro, and F. Seifert, Am. Mineral. 86, 348 (2001).

${ }^{91}$ M. A. Carpenter, in Transformation Processes in Minerals, edited by S. A. T. Redfern and M. A. Carpenter, Vol. 39 (Mineralogical Society of America, Washington DC, 2000), p. 35.

${ }^{92}$ R. L. Melcher and D. I. Bolef, Phys. Rev. 178, 864 (1969).

${ }^{93}$ M. A. Carpenter, R. E. A. McKnight, C. J. Howard, and K. S. Knight, Phys. Rev. B 82, 094101 (2010).

${ }^{94}$ T.-Y. Tan, B. J. Kennedy, Q. Zhou, C. D. Ling, W. Miiller, C. J. Howard, M. A. Carpenter, and K. S. Knight, Phys. Rev. B 85, 104107 (2012).

${ }^{95}$ E. K. H. Salje, B. Wruck, and H. Thomas, Z. Phys. B 82, 399 (1991).

${ }^{96}$ S. A. Hayward and E. K. H. Salje, J. Phys.: Condens. Matter 10, 1421 (1998).

${ }^{97}$ S. A. Hayward and E. K. H. Salje, Phase Transit. 68, 501 (1999).

${ }^{98}$ S. A. Hayward, S. A. T. Redfern, and E. K. H. Salje, J. Phys.: Condens. Matter 14, 10131 (2002).

${ }^{99}$ S. A. Hayward, F. D. Morrison, S. A. T. Redfern, E. K. H. Salje, J. F. Scott, K. S. Knight, S. Tarantino, A. M. Glazer, V. Shuvaeva, P. Daniel, M. Zhang, and M. A. Carpenter, Phys. Rev. B 72, 054110 (2005).

${ }^{100}$ D. J. Goossens, R. A. Robinson, and M. T. F. Telling, Physica B 352, 105 (2004).

${ }^{101}$ R. J. Harrison, S. A. T. Redfern, and E. K. H. Salje, Phys. Rev. B 69, 144101 (2004).
${ }^{102}$ J. Chrosch and E. K. H. Salje, J. Appl. Phys. 85, 722 (1999).

${ }^{103}$ W. T. Lee, E. K. H. Salje, L. Goncalves-Ferreira, M. Daraktchiev, and U. Bismayer, Phys. Rev. B 73, 214110 (2006).

${ }^{104}$ M. A. Carpenter and Z. Zhang, Geophys. J. Int. 186, 279 (2011).

${ }^{105}$ E. K. H. Salje, X. Ding, Z. Zhao, T. Lookman, and A. Saxena, Phys. Rev. B 83, 104109 (2011).

${ }^{106}$ J. A. Kilner, Philos. Mag. A 43, 1473 (1981).

${ }^{107}$ R. R. Becher, M. J. L. Sangster, and D. Strauch, J. Phys.: Condens. Matter 1, 7801 (1989).

${ }^{108}$ W. Schranz, P. Sondergeld, A. V. Kityk, and E. K. H. Salje, Phase Transit. 69, 61 (1999).

${ }^{109}$ A. V. Kityk, W. Schranz, P. Sondergeld, D. Havlik, E. K. H. Salje, J. F. Scott, and J. F. Scott, Europhys. Lett. 50, 41 (2000).

${ }^{110}$ A. V. Kityk, W. Schranz, P. Sondergeld, D. Havlik, E. K. H. Salje, and J. F. Scott, Phys. Rev. B 61, 946 (2000).

${ }^{111}$ V. V. Lemanov, S. A. Gridnev, and E. V. Ukhin, Phys. Solid State 44, 1106 (2002).

${ }^{112}$ R. J. Harrison and S. A. T. Redfern, Phys. Earth Planet. Inter. 134, 253 (2002).

${ }^{113}$ R. J. Harrison, S. A. T. Redfern, A. Buckley, and E. K. H. Salje, J. Appl. Phys. 95, 1706 (2004).

${ }^{114}$ W. Schranz, Phys. Rev. B 83, 094120 (2011).

${ }^{115}$ S. M. Shapiro, J. D. Axe, G. Shirane, and T. Riste, Phys. Rev. B 6, 4332 (1972).

${ }^{116}$ R. J. Birgeneau, J. K. Kjems, G. Shirane, and L. G. Van Uitert, Phys. Rev. B 10, 2512 (1974).

${ }^{117}$ M. A. Carpenter, C. J. Howard, B. J. Kennedy, and K. S. Knight, Phys. Rev. B 72, 024118 (2005)

${ }^{118}$ M. Wencka, S. Vrtnik, M. Jagodic, Z. Jaglicic, S. Turczynski, D. A. Pawlak, and J. Dolinsek, Phys. Rev. B 80, 224410 (2009).

${ }^{119}$ E. K. H. Salje and H. Zhang, Phase Transit. 82, 452 (2009).

${ }^{120}$ E. K. H. Salje, Chem. Phys. Chem. 11, 940 (2010).

${ }^{121}$ L. Goncalves-Ferreira, S. A. T. Redfern, E. Artacho, and E. K. H. Salje, Phys. Rev. Lett. 101, 097602 (2008).

${ }^{122}$ S. Van Aert, S. Turner, R. Delville, D. Schryvers, G. Van Tendeloo, and E. K. H. Salje, Adv. Mater. 24, 523 (2012).

${ }^{123}$ S. J. Pickart, H. A. Alperin, and R. Nathans, J. Phys. 25, 565 (1964). 